NBER WORKING PAPER SERIES

\title{
ESTIMATORS FOR PERSISTENT AND POSSIBLY NON-STATIONARY DATA WITH CLASSICAL PROPERTIES
}

\author{
Yuriy Gorodnichenko \\ Anna Mikusheva \\ Serena $\mathrm{Ng}$ \\ Working Paper 17424 \\ http://www.nber.org/papers/w17424
}

\author{
NATIONAL BUREAU OF ECONOMIC RESEARCH \\ 1050 Massachusetts Avenue \\ Cambridge, MA 02138 \\ September 2011
}

Mikusheva acknowledges financial support from the Castle-Krob Career Development Chair. Ng acknowledges financial support from the National Science Foundation (SES-0962431). The views expressed herein are those of the authors and do not necessarily reflect the views of the National Bureau of Economic Research.

NBER working papers are circulated for discussion and comment purposes. They have not been peerreviewed or been subject to the review by the NBER Board of Directors that accompanies official NBER publications.

(C) 2011 by Yuriy Gorodnichenko, Anna Mikusheva, and Serena Ng. All rights reserved. Short sections of text, not to exceed two paragraphs, may be quoted without explicit permission provided that full credit, including $\left({ }^{\circ}\right.$ notice, is given to the source. 
Estimators for Persistent and Possibly Non-Stationary Data with Classical Properties Yuriy Gorodnichenko, Anna Mikusheva, and Serena Ng

NBER Working Paper No. 17424

September 2011

JEL No. C22,C32,E27,E37,G17

\section{ABSTRACT}

This paper considers a moments based non-linear estimator that is root-T consistent and uniformly asymptotically normal irrespective of the degree of persistence of the forcing process. These properties hold for linear autoregressive models, linear predictive regressions, as well as certain non-linear dynamic models. Asymptotic normality is obtained because the moments are chosen so that the objective function is uniformly bounded in probability and that a central limit theorem can be applied.

Critical values from the normal distribution can be used irrespective of the treatment of the deterministic terms. Simulations show that the estimates are precise, and the t-test has good size in the parameter region where the least squares estimates usually yield distorted inference.

Yuriy Gorodnichenko

Department of Economics

549 Evans Hall \#3880

University of California, Berkeley

Berkeley, CA 94720-3880

and NBER

ygorodni@econ.berkeley.edu

Anna Mikusheva

MIT

amikushe@mit.edu

\author{
Serena $\mathrm{Ng}$ \\ Department of Economics \\ Columbia University \\ 440 W. 118 St. \\ International Affairs Building, MC 3308 \\ New York \\ NY 10027 \\ serena.ng@columbia.edu
}




\section{Introduction}

This paper considers a quasi-differencing (QD) framework that can yield $\sqrt{T}$ consistent and uniformly asymptotically normal estimators of autoregressions and multiple regressions when the predictors are persistent and possibly non-stationary. The approach can also be used to estimate dynamic stochastic general equilibrium (DSGE) models. The critical values are invariant to the presence of deterministic trends.

Let $\theta$ be an unknown parameter vector and let $\theta^{0}$ be its true value. We propose non-linear $\mathrm{QD}$ estimators that can generically be defined as

$$
\widehat{\theta}_{K}=\underset{\theta \in \Theta}{\operatorname{argmin}} \bar{g}(\theta)^{\prime} W_{T} \bar{g}(\theta),
$$

where $\bar{g}(\theta)$ is a $K \times 1$ vector of moments, $W_{T}$ is a $K \times K$ positive-definite matrix, and $\Theta$ is a bounded set containing values of $\theta{ }^{1}$ The basic premise of QD estimation is that for $\widehat{\theta}_{K}$ to have classical properties, $\bar{g}(\theta)$ needs to be uniformly bounded in probability and that a central limit theorem can be applied. To this end, $\bar{g}(\theta)$ is defined as the difference between the normalized autocovariances of the variables in the model and the data, all quasi-differenced at a persistence parameter that is to be estimated jointly with other parameters of the model. The normalization and quasi-differencing together provide a non-linear transformation of the autocovariances to result in estimators that are robust to possible non-stationarity in the data.

Achieving asymptotic normality without knowing when the exogenous process has an autoregressive unit root can be very useful in applied work because the answers to many macroeconomic questions are sensitive to assumptions about the nature of the trend and to whether the corresponding regressions are estimated in levels or in first-differences. The price to pay for practical simplicity and robustness is that the proposed estimators are $\sqrt{T}$ consistent rather than super-consistent when the regressors are truly non-stationary. While other asymptotically normal estimators robust to non-stationary regressors are available, they apply only to specific linear models. The QD estimation framework is general and can be used whenever the variables can be quasi-differenced in the way discussed below.

We establish uniform asymptotic normality of QD-based estimators in many different settings.

\footnotetext{
${ }^{1}$ The optimization is performed over an expanded neighborhood of the set of admissible values of $\theta$ so that the parameter of interest is not on the boundary of the support. For the $\operatorname{AR}(1)$ model, the admissible values are $(-1+\delta, 1]$ where $\delta>0$. We optimize over $\Theta=\left[C_{1}, C_{2}\right]$, where $C_{1}<-1+\delta<1<C_{2}$. When the context is clear, $\Theta$ will not be explicitly specified.
} 
Throughout, we use the notion of uniformity given in Mikusheva (2007a), who studied uniform coverage properties of various inference procedures for the AR(1) model.

Definition 1 A family of distributions $F_{\theta, T}^{(1)}(x)=P_{\theta, T}\left\{\xi_{1}<x\right\}$ is asymptotically approximated by (converges to) a family of distributions $F_{\theta}^{(2)}(x)=P_{\theta}\left\{\xi_{2}<x\right\}$ uniformly over $\theta \in \Theta$ if

$$
\lim _{T \rightarrow \infty} \sup _{\theta \in \Theta} \sup _{x}\left|F_{\theta, T}^{(1)}(x)-F_{\theta}^{(2)}(x)\right|=0 \text {. }
$$

In our analysis, $F_{\theta}^{(2)}(x)$ is in the family of Gaussian distributions.

The paper is structured as follows. Section 2 provides a rigorous analysis of the AR(1) model. Section 3 extends the analysis to $\mathrm{AR}(\mathrm{p})$ models, while Section 4 studies predictive regressions. Section 5 considers non-linear estimation of structural parameters. Simulations are presented in Section 6. The relation of QD estimation to other $\sqrt{T}$-consistent estimators is discussed in Section 7. All proofs are in the Appendix. As a matter of notation, the indicator function $\mathbb{I}(a)$ is one if $a$ is true and zero otherwise. We let $W(\cdot)$ be the standard Brownian motion and use $\Rightarrow$ to denote weak convergence.

\section{The $\operatorname{AR}(1)$ Model}

To systematically motivate the idea behind QD estimation, we begin with the simple AR(1) model with parameter $\alpha$ and whose true value is $\alpha^{0}$. For $t=1, \ldots, T$, the data are generated by

$$
y_{t}=\alpha^{0} y_{t-1}+\varepsilon_{t}, \quad y_{0}=0
$$

Hereafter, we let $\varepsilon_{t}$ be the deviation between the dependent variable and the conditional mean evaluated at the true parameter value, while $e_{t}$ is the deviation evaluated at an arbitrary value of the parameter vector. The error $\varepsilon_{t}$ does not need to be iid or Gaussian, but it cannot be conditional heteroscedastic or heteroscedastic.

Assumption A $\left(\varepsilon_{t}, \mathcal{F}_{t}\right)$ is a stationary ergodic martingale-difference sequence with conditional variance $E\left(\varepsilon_{t}^{2} \mid \mathcal{F}_{t-1}\right)=\sigma^{2}=\gamma_{0}$ and $E\left(\left(\varepsilon_{t}^{2}-\sigma^{2}\right)^{2} \mid \mathcal{F}_{t-1}\right)=\mu_{4}$. 
The least squares estimator $\widehat{\alpha}_{O L S}$ is defined as the solution to $\bar{g}(\alpha)=0$, where $\bar{g}(\alpha)=\frac{1}{T} \sum_{t=1}^{T} e_{t} y_{t-1}$ is the sample analog of the moment condition

$$
E g_{t}\left(\alpha^{0}\right)=E\left[\varepsilon_{t} y_{t-1}\right]=0
$$

with $e_{t}=\left(y_{t}-\alpha y_{t-1}\right)$. When $\alpha^{0}<1$, $\widehat{\alpha}_{O L S}$ is $\sqrt{T}$ consistent and asymptotically normal. While $\widehat{\alpha}_{O L S}$ is super-consistent at $\alpha^{0}=1$, its distribution is non-standard which makes inference difficult. In particular, the $t$-statistic for testing $\alpha^{0}=1$ is non-normal in finite samples and converges to the so-called Dickey-Fuller distribution. The issue of non-standard inference arises because of two problems. First, when $\alpha^{0}=1$, the sample moment evaluated at a value $\alpha \neq \alpha^{0}=1$ explodes, and second, the normalized sample moment evaluated at the true value does not obey a central limit theorem. Specifically, $\bar{g}(\alpha)=\frac{1}{T} \sum_{t=1}^{T} e_{t} y_{t-1}$ is stochastically unbounded, and $\frac{1}{T} \sum_{t=1}^{T} \varepsilon_{t} y_{t-1} \Rightarrow$ $\sigma^{2} \int_{0}^{1} W(r) d W(r)$, where $W(r)$ is the standard Brownian motion.

Our starting point is to resolve the second issue by exploiting the autocovariance structure of the errors. Specifically, for $j \geq 1$, it holds that

$$
E\left(\varepsilon_{t} \varepsilon_{t-j}\right)=0
$$

Furthermore, for all $\left|\alpha^{0}\right| \leq 1$ and $\varepsilon_{t}=y_{t}-\alpha^{0} y_{t-1}$, the population moment condition has a sample analog that obeys a central limit theorem:

$$
\sqrt{T}\left(\frac{1}{T} \sum_{t=j+1}^{T} \varepsilon_{t} \varepsilon_{t-j}-E\left(\varepsilon_{t} \varepsilon_{t-j}\right)\right) \Rightarrow \xi_{j} \sim N\left(0, \sigma^{4}\right) .
$$

Obviously, $\alpha^{0}$ is unknown and $\varepsilon_{t}$ is not observed. However, we can quasi-difference $y_{t}$ at some $\alpha$ and then optimize over all possible values of $\alpha$ by matching the sample autocovariances of the quasi-differenced data ${ }^{2}$

$$
\widehat{\gamma}_{j}(\alpha)=\frac{1}{T} \sum_{t=j+1}^{T} e_{t} e_{t-j}
$$

with those of the model evaluated under the assumption that $\alpha$ is the true value. Precisely, the model implied moments are

$$
\gamma_{j}(\alpha)=E_{\alpha} e_{t} e_{t-j}=\mathbb{I}(j=0) \sigma^{2},
$$

where $E_{\alpha}$ is the expectation taken under the (not necessarily correct) assumption that $\alpha$ is the true value. In cases when $\gamma_{j}(\alpha)$ is constant, the dependence of $\gamma_{j}$ on $\alpha$ can be suppressed. For

\footnotetext{
${ }^{2}$ To be more precise, we should write $\widehat{\gamma}_{j}\left(\alpha, \alpha^{0}, \sigma^{2}\right)$ because the data are generated under $\alpha^{0}$ and $\sigma^{2}$, and we quasi-difference the data at $\alpha$. For notational simplicity, the dependence of $\widehat{\gamma}_{j}$ on $\alpha^{0}$ and $\sigma^{2}$ are suppressed.
} 
example, in the $\operatorname{AR}(1)$ under consideration, $\gamma_{j}(\alpha)=0$ for all $j \geq 1$, and we may write $\gamma_{j}$ instead of $\gamma_{j}(\alpha)$. This is also true of the $\operatorname{AR}(\mathrm{p})$ and predictive regressions considered in the sections 3 and 4. However, in more complex models such as the one considered in Section $5, \gamma_{j}$ often depends on $\alpha$ in a complicated and analytically intractable way. For clarity, we keep the explicit dependence of $\gamma_{j}$ on $\alpha$ throughout.

Let $\bar{g}_{N Q D}(\alpha)=\left(\bar{g}_{0, N Q D}(\alpha), \ldots, \bar{g}_{K, N Q D}(\alpha)\right)^{\prime}$, where

$$
\bar{g}_{j, N Q D}(\alpha)=\widehat{\gamma}_{j}(\alpha)-\gamma_{j}(\alpha)
$$

be the difference between the model-implied and the sample autocovariance of $e_{t}$. The estimator is defined as

$$
\widehat{\alpha}_{K, N Q D}=\underset{\alpha}{\operatorname{argmin}} \bar{g}_{N Q D}(\alpha)^{\prime} W_{T} \bar{g}_{N Q D}(\alpha) .
$$

Although $\widehat{\alpha}_{K, N Q D}$ is a standard GMM estimator, viewing it from the perspective of a covariance structure estimator helps understand the analysis to follow. In standard covariance structure estimation where a typical element of $\bar{g}(\alpha)$ is $\bar{g}_{j}(\alpha)=\frac{1}{T} \sum_{t=1}^{T} y_{t} y_{t-1}-E_{\alpha} y_{t} y_{t-1}$, each sample autocovariance is a function of the data $y_{t}$ and hence depends on $\alpha^{0}$, but it does not depend on $\alpha$. In our $\bar{g}_{j, N Q D}(\alpha)$, both the sample and model moments depend on $\alpha$. It differs from the standard formulation of covariance structure estimation but it is necessary because $\sqrt{T}\left(\frac{1}{T} \sum_{t=1}^{T} y_{t} y_{t-1}-\right.$ $\left.E_{\alpha} y_{t} y_{t-1}\right)$ does not obey a central limit theorem at $\alpha=\alpha^{0}=1$.

The NQD solves one of the two problems inherent in least squares estimation by making $\sqrt{T} \bar{g}_{j, N Q D}\left(\alpha^{0}\right)$ asymptotically normal for all $\left|\alpha^{0}\right| \leq 1$. However, the estimator still has non-standard properties because $\widehat{\gamma}_{j}(\alpha)$ is stochastically unbounded when $\alpha^{0}=1$ and $\alpha \neq 1$. Thus the moment $\bar{g}_{j, N Q D}(\alpha)$ explodes at $\alpha \neq \alpha^{0}$ when $\alpha^{0}$ is unity or in the neighborhood of one. To resolve this problem, suppose $\gamma_{0}$ is known and define

$$
\begin{aligned}
\bar{g}_{j, F Q D}(\alpha) & =\bar{g}_{j, N Q D}(\alpha)-\bar{g}_{0, N Q D}(\alpha) \\
& =\left(\widehat{\gamma}_{j}(\alpha)-\widehat{\gamma}_{0}(\alpha)\right)-\left(\gamma_{j}(\alpha)-\gamma_{0}\right), \\
\widehat{\alpha}_{K, F Q D} & =\underset{\alpha}{\operatorname{argmin}} \bar{g}_{F Q D}(\alpha)^{\prime} W_{T} \bar{g}_{F Q D}(\alpha) .
\end{aligned}
$$

Obviously, $\bar{g}_{j, F Q D}\left(\alpha^{0}\right)$ obeys a central limit theorem. More important is that normalizing $\widehat{\gamma}_{j}(\alpha)$ by $\widehat{\gamma}_{0}(\alpha)$ and $\gamma_{j}(\alpha)$ by $\gamma_{0}$ yield

$$
\bar{g}_{j, F Q D}(\alpha)=\frac{1}{T} \sum_{t=j+1}^{T} e_{t}\left(e_{t-j}-e_{t}\right)+\gamma_{0}
$$


As shown in Lemma A-2 of the Appendix, $\widehat{\gamma}_{j}(\alpha)-\widehat{\gamma}_{0}(\alpha)$ is bounded in probability in the limit even when $\alpha^{0}=1$ and $\alpha \neq \alpha^{0}$. Because $\bar{g}_{j, F Q D}(\alpha)$ is uniformly bounded in probability for all values over which $\alpha$ is optimized and $\alpha^{0} \in(1-\delta, 1]$, the FQD has very different properties from the NQD in the local-to-unity framework.

To gain insight about the importance of normalization, we let $W_{T}$ be an identity matrix as it does not affect uniformity arguments and this simplifying assumption makes it possible to obtain useful closed form expressions.

Proposition 1 Let $y_{t}$ be generated as in equation (2) with error terms satisfying Assumption A. Assume that $W_{T}$ is a $K \times K$ identity matrix.

i. Let $n \in\{1,2\}$ be the number of local minima in the optimization problem $\min _{\alpha} \sum_{k=1}^{K} \bar{g}_{k, N Q D}^{2}(\alpha)$. In the local-to-unity framework in which $\alpha^{0}=1+c / T$ with $c \leq 0, \widehat{\alpha}_{K, N Q D}$ is super-consistent and

$$
\left(\begin{array}{c}
\mathbb{I}\{n=2\} \cdot T^{3 / 2}\left(\widehat{\alpha}_{K, N Q D}-\alpha^{0}\right)^{2} \\
\mathbb{I}\{n=1\} \cdot T\left(\widehat{\alpha}_{K, N Q D}-\alpha^{0}\right)
\end{array}\right) \Rightarrow\left(\begin{array}{c}
\frac{-\xi}{\int_{0}^{1} J_{c}^{2}(s) d s} \mathbb{I}\{\xi<0\} \\
\frac{1+2 \int_{0}^{1} J_{c}(s) d W(s)}{2 \int_{0}^{1} J_{c}^{2}(s) d s} \mathbb{I}\{\xi>0\}
\end{array}\right)
$$

where $J_{c}$ is an Ornstein-Uhlenbeck process generated by the Brownian motion $W$ that is independent of $\xi \sim N(0,1 / K)$.

ii. Let $\gamma_{0}$ be the true value of $\sigma^{2}$. For any fixed $K>1$, the estimator $\widehat{\alpha}_{K, F Q D}$ is consistent. Furthermore, uniformly over $-1+\delta<\alpha^{0} \leq 1$ :

$$
\sqrt{T}\left(\widehat{\alpha}_{K, F Q D}-\alpha^{0}\right) \Rightarrow N\left(0, \sigma_{K, F Q D}^{2}\right), \quad \text { where } \quad \sigma_{K, F Q D}^{2}=\frac{\left(\sum_{k=1}^{K}\left(\alpha^{0}\right)^{(k-1)}\right)^{2} \frac{\mu_{4}}{\sigma^{4}}+\sum_{k=1}^{K}\left(\alpha^{0}\right)^{2(k-1)}}{\left(\sum_{k=1}^{K}\left(\alpha^{0}\right)^{2(k-1)}\right)^{2}} .
$$

The NQD estimator is the basis of the estimators we subsequently investigate. It is consistent and has a data dependent convergence rate. Since the objective function is a polynomial of the fourth order, there are multiple solutions. If the realization of data is such that there is a unique minimum to the optimization problem, the convergence rate is $T$. If there are two minima, a slower convergence rate of $T^{3 / 4}$ is obtained. In either case, the distribution of $\widehat{\alpha}_{K, N Q D}$ is not asymptotically normal because $\bar{g}_{N Q D}(\alpha)$ is not well behaved for all values of $\alpha^{0}$. However, the problematic term that frustrates a quadratic expansion of $\widehat{\gamma}_{j}(\alpha)$ around $\alpha^{0}$ is asymptotically collinear with the corresponding term in $\widehat{\gamma}_{0}(\alpha)$ in the local-to-unity framework. Normalizing each $\widehat{\gamma}_{j}(\alpha)$ by $\widehat{\gamma}_{0}(\alpha)$ and 
$\gamma_{j}(\alpha)$ by $\gamma_{0}$ results in an FQD estimator whose asymptotic distribution is normal uniformly over $\alpha^{0} \in(-1+\delta, 1]$. When $K>1$, the FQD objective function has only one minimum asymptotically. ${ }^{3}$

The properties for $\widehat{\alpha}_{K, F Q D}$ are stated assuming the true value of $\sigma^{2}$ is known. The reason why $\gamma_{0}=\sigma^{2}$ is not freely estimated along with $\alpha$ is that doing so would yield multiple solutions. The objective function is zero not only at the true solution $\alpha=\alpha^{0}, \sigma^{2}=\gamma_{0}$, but also at $\alpha=1 / \alpha^{0}, \sigma^{2}=$ $\gamma_{0} /\left(\alpha^{0}\right)^{2}$. Without additional information, the FQD cannot uniquely identify $\alpha$ and $\sigma^{2}$.

In practice, the true value of $\sigma^{2}$ is not known, and the FQD estimator is infeasible. This can be overcome by finding another moment that can identify $\sigma^{2}$. Let $\theta=\left(\alpha, \sigma^{2}\right)$ and consider

$$
\bar{g}_{Q D}(\theta)=\left(\begin{array}{c}
s^{2}-\sigma^{2} \\
\widehat{\gamma}_{1}(\alpha)-\widehat{\gamma}_{0}(\alpha)-\left(\gamma_{1}(\alpha)-s^{2}\right) \\
\vdots \\
\widehat{\gamma}_{K}(\alpha)-\widehat{\gamma}_{0}(\alpha)-\left(\gamma_{K}(\alpha)-s^{2}\right)
\end{array}\right) .
$$

where $s^{2}=\frac{1}{T} y^{\prime} M y$, and $M=I_{T}-z\left(z^{\prime} z\right)^{-1} z^{\prime}$ is the matrix that projects onto the space orthogonal to $z$ with $z_{t}=y_{t-1}$. Observe that the first component of $\bar{g}_{Q D}(\theta)$ is $s^{2}-\sigma^{2}$ or equivalently $s^{2}-\gamma_{0}(\alpha)$, but not $s^{2}-\widehat{\gamma}_{0}(\alpha)$. Thus, $\bar{g}_{Q D}(\theta)$ is not a linear transformation of $\bar{g}_{N Q D}(\theta)$. Using $s^{2}-\widehat{\gamma}_{0}(\alpha)$ in the first entry would result in an estimator with the same non-standard properties like $\widehat{\alpha}_{N Q D}$.

Let

$$
\widehat{\theta}_{K, Q D}=\left(\widehat{\alpha}_{K, Q D}, \widehat{\sigma}_{K, Q D}^{2}\right)=\underset{\theta}{\operatorname{argmin}} \bar{g}_{Q D}(\theta)^{\prime} W_{T} \bar{g}_{Q D}(\theta)
$$

Proposition 2 Let $W_{T}$ be a $(K+1) \times(K+1)$ identity matrix. For any fixed $K>1$, the estimator $\widehat{\alpha}_{K, Q D}$ is consistent, and the following convergence holds uniformly over $-1+\delta<\alpha^{0} \leq 1$ :

$$
\sqrt{T}\left(\widehat{\alpha}_{K, Q D}-\alpha^{0}\right) \Rightarrow N\left(0, \sigma_{K, Q D}^{2}\right), \text { where } \sigma_{K, Q D}^{2}=\frac{\sum_{k=1}^{K}\left(\alpha^{0}\right)^{2(k-1)}}{\left(\sum_{k=1}^{K}\left(\alpha^{0}\right)^{2(k-1)}\right)^{2}} .
$$

Estimator $\widehat{\alpha}_{K, Q D}$ can also be implemented as a two-step estimator in which $s^{2}$ is first obtained, and its value would then be used as $\gamma_{0}$ in the moment function $\bar{g}_{F Q D}(\alpha)=\left(\bar{g}_{1, F Q D}(\alpha), \ldots, \bar{g}_{K, F Q D}(\alpha)\right)^{\prime}$.

The asymptotic variance of $\widehat{\alpha}_{K, Q D}$ takes into account the sampling uncertainty of $s^{2}$. The surprising aspect of Proposition 2 is that $\widehat{\alpha}_{K, Q D}$ does not have an inflated variance as is typical of two-step

\footnotetext{
${ }^{3}$ When the NQD has two local minima, it does not matter which one is chosen as they are asymptotically symmetric around the true value. This was why we state our result as $T^{3 / 2}\left(\widehat{\alpha}_{K, N Q D}-\alpha^{0}\right)^{2}$ rather $\operatorname{than} T^{3 / 4}\left(\widehat{\alpha}_{K, N Q D}-\alpha^{0}\right)$. If $K=1$, the FQD objective function has 2 minima, only one of which is consistent for $\alpha^{0}$.
} 
estimators. Instead, the estimator is more efficient than $\widehat{\alpha}_{K, F Q D}$ that has a known $\sigma^{2}$. Pierce (1982) showed in a framework for stationary data that using estimated values of nuisance parameters can yield statistics with smaller variance than if the nuisance parameters were known. This somewhat paradoxical result was also reported by Prokhorov and Schmidt (2009) and Han and Kim (2011) for GMM estimators. Our results suggest that this feature may also arise in the local-to-unity framework.

The closed form expression for the asymptotic variance of $\widehat{\alpha}_{K, Q D}$ in Proposition 2 was obtained under the assumption that $W_{T}$ is an identity matrix. For an arbitrary positive-definite weighting matrix, the asymptotic variance of $\widehat{\alpha}_{K, Q D}$ is the (1,1)-th element of asymptotic variance matrix

$$
\operatorname{Avar}\left(\widehat{\theta}_{K, Q D}\right)=\left(G^{0 \prime} W G^{0}\right)^{-1} G^{0 \prime} W S^{0} W G^{0}\left(G^{0 \prime} W G^{0}\right)^{-1}
$$

where $W, G^{0}$ and $S^{0}$ are the probability limits of $W_{T}$, the derivative of $\bar{g}_{Q D}(\theta)$ with respect to $\theta$ evaluated at $\theta^{0}$, and the asymptotic variance of $\bar{g}_{Q D}\left(\theta^{0}\right)$, respectively. The asymptotic variance can thus be estimated as though the GMM estimator were developed in the stationary framework under regularity conditions such as those given in Newey and McFadden (1994). In theory, more efficient estimates can be obtained if $W_{T}$ is an optimal weighting matrix. However, it has been documented in Abowd and Card (1989) and Altonji and Segal (1996) that an optimal weighting matrix may not be desirable for covariance structure estimation for empirically relevant sample sizes.

The key to the classical properties of $\widehat{\alpha}_{K, Q D}$ is the ability to exploit the autocovariance properties of the quasi-differenced data in an appropriate way. Quasi-differencing has a long tradition in econometrics and underlies GLS estimation, see Phillips and Xiao (1998). Canjels and Watson (1997) and Phillips and Lee (1996) found that quasi-differencing gives more precise estimates of trend parameters when the errors are highly persistent. Pesavento and Rossi (2006) suggest that for such data, quasi-differencing can improve the coverage of impulse response functions. In both studies, the data are quasi-differenced at $\alpha=\bar{\alpha}$ which is fixed at the value suggested by the localto-unity framework. In contrast, the FQD and QD simultaneously estimate this parameter and use the normalized autocovariances of the quasi-differenced data for estimation. Notably, both the FQD and the QD have classical properties that hold even in the presence of deterministic terms. Consider data generated as

$$
\begin{aligned}
& y_{t}=d_{t}+x_{t}, \\
& x_{t}=\alpha^{0} x_{t-1}+\varepsilon_{t} .
\end{aligned}
$$


The deterministic terms are captured by $d_{t}=\sum_{j=0}^{r} \psi_{j} t^{j}$ where $r$ is the order of the deterministic trend function. In the intercept-only case, $d_{t}=\psi_{0}$, and in the linear trend case, $d_{t}=\psi_{0}+\psi_{1} t$. Once the parameters of the trend function are consistently estimated, QD estimation proceeds by replacing $y_{t}$ with demeaned or de-trended data, $\widehat{x}_{t}=y_{t}-\widehat{d}_{t}$. Let $\widehat{e}_{t}=\widehat{x}_{t}-\alpha \widehat{x}_{t-k}$. The sample autocovariances can be constructed as

$$
\widehat{\gamma}_{k}(\alpha)=\frac{1}{T} \sum_{t=k+1}^{T} \widehat{e}_{t} \widehat{e}_{t-k} .
$$

Demeaning and de-trending do not affect the asymptotic distribution of the QD. ${ }^{4}$

The practical appeal of QD estimation is that asymptotic normality permits standard inference. The usual critical values of \pm 1.96 and \pm 1.64 can be used for two-tailed tests at the 5 and 10 percent significance levels, respectively. We will see in simulations that the size of tests and the coverage of the confidence sets based on the asymptotic normality of $\widehat{\alpha}_{K, Q D}$ are stable over the parameter set $\alpha^{0} \in(-1+\delta, 1]$. The cost of imposing the stronger assumption of conditional homoscedasticity seems well justified.

To recapitulate, the proposed QD estimation of the AR(1) model is based on two simple premises: first, that for all $j \geq 1, E\left(\varepsilon_{t} \varepsilon_{t-j}\right)=0$ and its sample analog obeys a central limit theorem, and, second, that the objective function is uniformly bounded in probability for all values of $\alpha$ and $\alpha^{0}$. The idea can be used whenever the variables can be quasi-differenced to form suitably normalized moment conditions that satisfy these two properties. The next two sections consider the AR(p) model and predictive regressions, respectively. We then show that the quasi-differenced variables can be serially correlated and that the QD framework can be used in non-linear estimations.

\section{$3 \quad \operatorname{AR}(\mathbf{p})$ Models}

Consider the data generating process

$$
y_{t}=\alpha^{0} y_{t-1}+\sum_{j=1}^{p-1} b_{j}^{0} \Delta y_{t-j}+\varepsilon_{t} .
$$

\footnotetext{
${ }^{4}$ De-trending does not affect the asymptotic distribution of FQD, but the $J_{c}$ in the distribution of the NQD estimator will depend on $d_{t}$. In the intercept only case, one should use the de-meaned Ornstein-Uhlenbeck process $\bar{J}_{c}(r)=J_{c}(r)-\int_{0}^{1} J_{c}(s) d s$. In the linear trend case, the de-trended process is $\widetilde{J}_{c}(r)=J_{c}(r)-\int_{0}^{1}(4-6 s) J_{c}(s) d s-$ $r \int_{0}^{1}(12-6 s) J(s) d s$.
} 
Let $\beta=\left(\alpha, b_{1}, \ldots, b_{p-1}\right)$ be a $p \times 1$ parameter vector of interest. The true parameter vector is denoted $\beta^{0}$, and the correct lag length is denoted $p$. Let $\left|\lambda_{1}\right| \leq\left|\lambda_{2}\right| \ldots \leq\left|\lambda_{p}\right|$ be defined implicitly by the identity

$$
1-\alpha L-\sum_{j=1}^{p-1} b_{j} L^{j}(1-L)=\left(1-\lambda_{1} L\right) \ldots\left(1-\lambda_{p} L\right) .
$$

We restrict the parameter set in such a way that the $p-1$ smallest roots do not exceed $\delta$ in absolute value for some fixed $0<\delta<1$. If the largest root exceeds $\delta$ in absolute value, then it is positive and not larger than 1.

Definition 2 The parameter set $\mathfrak{R}_{\delta}$ consists of all $\beta$ such that the corresponding roots satisfy the following two conditions: (i) $\left|\lambda_{p-1}\right|<\delta$, (ii) if $\lambda_{p} \in \mathbb{R}$, then $-\delta \leq \lambda_{p} \leq 1 .^{5}$

Define the quasi-differenced series $e_{t}$ by

$$
e_{t}=y_{t}-\alpha y_{t-1}-\sum_{j=1}^{p-1} b_{j} \Delta y_{t-j}
$$

Obviously, $e_{t}=\varepsilon_{t}$ is white noise when $\beta=\beta^{0}$, but $e_{t}$ is in general serially correlated. Thus, as in the $\operatorname{AR}(1)$ model, the model-implied autocovariances satisfy

$$
\gamma_{j}(\beta)=E_{\beta}\left(e_{t} e_{t-j}\right)=0, \quad j \geq 1 \quad \forall \beta \in \mathfrak{R}_{\delta}
$$

with $\gamma_{0}(\beta)=\sigma^{2}$. The sample autocovariances of $e_{t}$ are

$$
\widehat{\gamma}_{j}(\beta)=\frac{1}{T} \sum_{t=j+p+1}^{T} e_{t} e_{t-j}
$$

Let $s^{2}=\frac{1}{T} y^{\prime} M y$ where $M$ projects onto the space orthogonal to the one spanned by $X_{t}=$ $\left(y_{t-1}, \Delta y_{t-1}, \ldots, \Delta y_{t-p+1}\right)^{\prime}, t=1, \ldots, T$. Let $\gamma_{0}$ be the true value of $\sigma^{2}$. Define

$$
\widehat{\beta}_{K, F Q D}=\underset{\beta}{\operatorname{argmin}} \bar{g}_{F Q D}(\beta)^{\prime} W_{T} \bar{g}_{F Q D}(\beta)
$$

where

$$
\bar{g}_{F Q D}(\beta)=\left(\begin{array}{c}
\bar{g}_{1, F Q D}(\beta) \\
\vdots \\
\bar{g}_{K, F Q D}(\beta)
\end{array}\right)=\left(\begin{array}{c}
\widehat{\gamma}_{1}(\beta)-\widehat{\gamma}_{0}(\beta)-\left(\gamma_{1}(\beta)-\gamma_{0}\right) \\
\vdots \\
\widehat{\gamma}_{K}(\beta)-\widehat{\gamma}_{0}(\beta)-\left(\gamma_{K}(\beta)-\gamma_{0}\right)
\end{array}\right) .
$$

\footnotetext{
${ }^{5}$ The optimization in equation (1) is done over a bounded set that includes a neighborhood of $\mathfrak{R}_{\delta}$ in order to avoid the boundary problem.
} 
Define

$$
\left(\widehat{\beta}_{K, Q D}, \widehat{\sigma}_{K, Q D}^{2}\right)=\underset{\beta, \sigma^{2}}{\operatorname{argmin}} \bar{g}_{Q D}\left(\beta, \sigma^{2}\right)^{\prime} W_{T} \bar{g}_{Q D}\left(\beta, \sigma^{2}\right),
$$

where

$$
\bar{g}_{Q D}\left(\beta, \sigma^{2}\right)=\left(\begin{array}{c}
\bar{g}_{0, Q D}(\beta) \\
\bar{g}_{1, Q D}(\beta) \\
\vdots \\
\bar{g}_{K, Q D}(\beta)
\end{array}\right)=\left(\begin{array}{c}
\widehat{\gamma}_{1}(\beta)-\widehat{\gamma}_{0}(\beta)-\left(\gamma_{1}(\beta)-s^{2}\right) \\
\vdots \\
\widehat{\gamma}_{K}(\beta)-\widehat{\gamma}_{0}(\beta)-\left(\gamma_{K}(\beta)-s^{2}\right)
\end{array}\right)
$$

Proposition 3 Let $y_{t}$ be generated as in equation (10) with error terms satisfying Assumption A. Let $a_{k}=E\left[\left(X_{t+k}+X_{t-k}-2 X_{t}\right) \varepsilon_{t}\right]$ and $G=\left(\sum_{k=1}^{K} a_{k} a_{k}^{\prime}\right)^{-1}$. For any fixed $K>p>1$, the estimators $\widehat{\beta}_{K, Q D}$ and $\widehat{\beta}_{K, F Q D}$ are consistent. Furthermore, when $W_{T}$ is an identity weighting matrix, the following results hold uniformly over $\beta^{0} \in \mathfrak{R}_{\delta}$ :

(i) $\sqrt{T}\left(\widehat{\beta}_{K, F Q D}-\beta^{0}\right) \Rightarrow N\left(0, \Sigma_{K, F Q D}\right)$, where $\Sigma_{K, F Q D}=\sigma^{4} G+\mu_{4} G\left(\sum_{k=1}^{K} a_{k}\right)\left(\sum_{k=1}^{K} a_{k}\right)^{\prime} G$;

(ii) $\sqrt{T}\left(\widehat{\beta}_{K, Q D}-\beta^{0}\right) \Rightarrow N\left(0, \Sigma_{K, Q D}\right)$, where $\Sigma_{K, Q D}=\sigma^{4} G$.

The proof is a generalization of Propositions 1 and 2. A sketch of the arguments is as follows. From the definition that $e_{t}(\beta)=\varepsilon_{t}+\left(\beta^{0}-\beta\right)^{\prime} X_{t}$, we have

$$
\widehat{\gamma}_{j}(\beta)=\frac{1}{T} \sum_{t=j+p+1}^{T} \varepsilon_{t} \varepsilon_{t-j}+\left(\beta^{0}-\beta\right)^{\prime} \frac{1}{T} \sum_{t=j+p+1}^{T}\left(X_{t} \varepsilon_{t-j}+X_{t-j} \varepsilon_{t}\right)+\left(\beta^{0}-\beta\right)^{\prime} \frac{1}{T} \sum_{t=j+p+1}^{T} X_{t} X_{t-j}^{\prime}\left(\beta^{0}-\beta\right) .
$$

The moment function can be rewritten as

$$
\bar{g}_{j, F Q D}(\beta)=A_{j, F Q D}+\left(\beta^{0}-\beta\right)^{\prime} B_{j, F Q D}+\left(\beta^{0}-\beta\right)^{\prime} C_{j, F Q D}\left(\beta^{0}-\beta\right) .
$$

The thrust of the proof is to show that for each $1 \leq j \leq K$ uniformly over $\mathfrak{R}_{\delta}$,

$$
\begin{aligned}
& A_{j, F Q D}=\left(\frac{1}{T} \sum_{t=j+p+1}^{T} \varepsilon_{t} \varepsilon_{t-j}-\gamma_{j}(\beta)\right)-\left(\frac{1}{T} \sum_{t=p+1}^{T} \varepsilon_{t}^{2}-\gamma_{0}\left(\beta^{0}\right)\right)=O_{p}\left(T^{-1 / 2}\right), \\
& B_{j, F Q D}=\frac{1}{T} \sum_{t=j+p+1}^{T}\left(X_{t} \varepsilon_{t-j}+X_{t-j} \varepsilon_{t}-2 X_{t} \varepsilon_{t}\right) \rightarrow^{p} a_{j}, \\
& C_{j, F Q D}=\frac{1}{2 T} \sum_{t=j+p+1}^{T}\left(X_{t} X_{t-j}^{\prime}+X_{t-j} X_{t}^{\prime}-2 X_{t} X_{t}^{\prime}\right)=O_{p}(1) .
\end{aligned}
$$


Equations (11)-(13) imply that the function $\bar{g}_{j, F Q D}(\beta)$ is bounded in probability uniformly for all $\beta$ in the optimization set and $\beta_{0} \in \mathfrak{R}_{\delta}$. It also follows from equations (11)-(13) that

$$
\frac{\partial \bar{g}_{j, F Q D}}{\partial \beta}\left(\widehat{\beta}_{K, F Q D}\right) \rightarrow^{p} a_{j}
$$

and

$$
\sqrt{T} \bar{g}_{k, F Q D}\left(\widehat{\beta}_{K, F Q D}\right)=\sqrt{T} A_{k, F Q D}+a_{k}^{\prime} \sqrt{T}\left(\widehat{\beta}_{K, F Q D}-\beta^{0}\right)+o_{p}(1)
$$

The first order condition for the optimization problem implies:

$$
\sqrt{T}\left(\widehat{\beta}_{K, F Q D}-\beta^{0}\right)=G^{-1} \sum_{j=1}^{K} \sqrt{T} A_{j, F Q D} a_{j}+o_{p}(1)
$$

In view of equation (4), $\sqrt{T} A_{j, F Q D}=\sqrt{T} \bar{g}_{j, F Q D}\left(\beta^{0}\right) \Rightarrow \xi_{j}-\xi_{0}$ uniformly over $\mathfrak{R}_{\delta}$, part (i) of the proposition follows. Part (ii) uses a similar argument with one exception: $A_{j, Q D}=A_{j, F Q D}+$ $s^{2}-\sigma^{2}=\frac{1}{T} \sum_{t=j+1}^{T} \varepsilon_{t} \varepsilon_{t-j}$. As in the $\operatorname{AR}(1)$ case, $\widehat{\beta}_{K, Q D}$ has a smaller variance than $\widehat{\beta}_{K, F Q D}$. Furthermore, one can use other weighting matrices in the estimation. The asymptotic variance of $\widehat{\beta}_{K, Q D}$ can be computed from the expression given in equation (8).

\section{Predictive Regressions}

Consider the predictive regression with scalar predictor $x_{t-1}$ :

$$
\begin{aligned}
& y_{t}=\beta^{0} x_{t-1}+\varepsilon_{y t} \\
& x_{t}=\alpha^{0} x_{t-1}+\varepsilon_{x t} .
\end{aligned}
$$

If $\alpha^{0}=1$, then $\left(1 ;-\beta^{0}\right)$ is a co-integrating vector, and ordinary least squares provide superconsistent estimates but inference is non-standard. Unfortunately, the finite sample distribution of $\widehat{\beta}_{O L S}$ is not well approximated by the normal distribution if $x_{t}$ is highly persistent. The challenge is how to conduct inference robust to the dynamic properties of $x_{t}$. Let $\varepsilon_{t}=\left(\varepsilon_{y t}, \varepsilon_{x t}\right)^{\prime}$ be a martingaledifference sequence with $E\left(\varepsilon_{t} \varepsilon_{t}^{\prime} \mid \mathcal{F}_{t-1}\right)=\Omega^{0}=\left(\begin{array}{ll}\sigma_{y y} & \sigma_{y x} \\ \sigma_{y x} & \sigma_{x x}\end{array}\right)$. Consider quasi-differencing the data at $\theta=(\beta, \alpha)$ to obtain

$$
\begin{aligned}
& e_{y t}=y_{t}-\beta x_{t-1}, \\
& e_{x t}=x_{t}-\alpha x_{t-1} .
\end{aligned}
$$


Now $Y_{t}=\theta^{0} x_{t-1}+\varepsilon_{t}$ where $Y_{t}=\left(\begin{array}{c}y_{t} \\ x_{t}\end{array}\right)$ and $\theta^{0}=\left(\beta^{0}, \alpha^{0}\right)^{\prime}$. Let $e_{t}=\left(\begin{array}{c}e_{y t} \\ e_{x t}\end{array}\right)$. Then

$$
e_{t}=\left(\theta^{0}-\theta\right) x_{t-1}+\varepsilon_{t}
$$

Let $\Gamma_{j}(\theta)=E_{\theta}\left(e_{t} e_{t-j}^{\prime}\right)$ where $E_{\theta}$ is the expectation taken under the assumption that $\theta$ is the true value. The model implies

$$
\begin{aligned}
& \Gamma_{j}(\theta)=0, \quad j \neq 0, \\
& \Gamma_{0}(\theta)=\Omega .
\end{aligned}
$$

The sample autocovariance at lag $j$ is

$$
\widehat{\Gamma}_{j}(\theta)=\frac{1}{T} \sum_{t=j+1}^{T} e_{t} e_{t-j}^{\prime} .
$$

A central limit theorem applies to $\sqrt{T} \widehat{\Gamma}_{j}\left(\theta^{0}\right)$. Evaluating $\Gamma_{0}$ at the true value of $\Omega$ and letting $S=\frac{1}{T} Y^{\prime} M Y, M=I_{T}-z\left(z^{\prime} z\right)^{-1} z^{\prime}, z_{t}=x_{t-1}$, we can define, for $j=1, \ldots, K$ :

$$
\bar{g}_{j, F Q D}(\theta)=\operatorname{vec}\left(\widehat{\Gamma}_{j}(\theta)-\widehat{\Gamma}_{0}(\theta)-\left(\Gamma_{j}(\theta)-\Gamma_{0}\right)\right) .
$$

Let $\bar{g}_{F Q D}(\theta)=\left(\bar{g}_{1, F Q D}(\theta)^{\prime}, \ldots, \bar{g}_{K, F Q D}(\theta)^{\prime}\right)^{\prime}$. The FQD estimator is

$$
\widehat{\theta}_{K, F Q D}=\arg \min _{\theta} \bar{g}_{F Q D}(\theta)^{\prime} W_{T} \bar{g}_{F Q D}(\theta)
$$

Analogously, let $\bar{g}_{Q D}(\theta, \Omega)=\left(\bar{g}_{0, Q D}(\theta, \Omega)^{\prime}, \ldots, \bar{g}_{K, Q D}(\theta, \Omega)^{\prime}\right)^{\prime}$ where

$$
\begin{aligned}
\bar{g}_{j, Q D}(\theta, \Omega) & =\operatorname{vec}\left(\widehat{\Gamma}_{j}(\theta)-\widehat{\Gamma}_{0}(\theta)-\left(\Gamma_{j}(\theta)-S\right)\right), \quad j \geq 1 \\
\bar{g}_{0, Q D}(\theta, \Omega) & =\operatorname{vech}(S-\Omega) .
\end{aligned}
$$

Define the QD estimator as

$$
\left(\widehat{\theta}_{K, Q D}, \widehat{\Omega}_{K, Q D}\right)=\arg \min _{\theta, \Omega} \bar{g}_{Q D}(\theta, \Omega)^{\prime} W_{T} \bar{g}_{Q D}(\theta, \Omega)
$$

Proposition 4 Suppose that the data are generated according to formulas (14a) and (14b). Suppose also that error terms are stationary martingale-difference sequence with $E\left(\varepsilon_{t} \varepsilon_{t}^{\prime} \mid \mathcal{F}_{t-1}\right)=\Omega^{0}$ and finite four moments. Define $a_{j}=E\left[x_{t-1}\left(\varepsilon_{t-j}-\varepsilon_{t}\right)\right]$. Then for any fixed $K>1$, the estimators $\widehat{\theta}_{K, F Q D}$ and $\widehat{\theta}_{K, Q D}$ are consistent. Furthermore, when $W_{T}$ is an identity matrix, the following asymptotic results hold uniformly over all possible values of $\beta$, and uniformly over all possible values of $\alpha \in(-1+\delta, 1]$ : 
(i) Let $\Gamma_{0}=\Omega^{0}$. Then $\sqrt{T}\left(\widehat{\theta}_{K, F Q D}-\theta^{0}\right) \Rightarrow N\left(0, \Sigma_{K, F Q D}\right)$, where

$$
\Sigma_{K, F Q D}=\left(\frac{1}{\sum_{k=1}^{K} a_{k}^{\prime} a_{k}}\right)^{2} \sum_{k=1}^{K}\left(a_{k}^{\prime} \Omega^{0} a_{k}\right) \Omega^{0}+\left(\frac{1}{\sum_{k=1}^{K} a_{k}^{\prime} a_{k}}\right)^{2} E\left[\left(\varepsilon_{t}^{\prime} \sum_{k=1}^{K} a_{j}\right)^{2} \varepsilon_{t} \varepsilon_{t}^{\prime}\right] ;
$$

(ii) $\sqrt{T}\left(\widehat{\theta}_{K, Q D}-\theta^{0}\right) \Rightarrow N\left(0, \Sigma_{K, Q D}\right)$ where

$$
\Sigma_{K, Q D}=\left(\frac{1}{\sum_{k=1}^{K} a_{k}^{\prime} a_{k}}\right)^{2} \sum_{k=1}^{K}\left(a_{k}^{\prime} \Omega^{0} a_{k}\right) \Omega^{0} .
$$

As in the case of autoregressions, the FQD moments alone cannot globally identify both $\theta$ and $\Omega$. Thus, the properties of $\widehat{\theta}_{K, F Q D}$ are stated by evaluating $\Omega$ at the true value of $\Omega^{0}$. Proposition 4 shows that $\widehat{\theta}_{K, Q D}$ has classical properties both in the stationary and the local-to-unity framework and is more efficient than the estimator $\widehat{\theta}_{K, F Q D}$ that uses the known $\Omega$. The QD can be implemented as a sequential estimator in which the covariance matrix is computed for shocks obtained from two least squares regressions: one by regressing $y_{t}$ on $x_{t}$ to get $\widehat{e}_{y t}$, and another autoregression in $x_{t}$ to obtain $\widehat{e}_{x t}$.

Proposition 4 has useful implications for applied work because there does not exist an estimator that is robust to the persistent properties of the predictors. The approach of Jansson and Moreira (2006) relies on model-specific conditional critical values and, in any event, their inference procedure does not yield an estimator per se. In contrast, the QD estimator is simple and robust.

The predictive regression can be generalized to accommodate stationary and pre-determined regressors, $z_{t}$. Suppose the data generating process is

$$
\begin{aligned}
& y_{t}=x_{t-1} \beta^{0}+z_{t} \gamma^{0}+\varepsilon_{y t} \\
& x_{t}=\alpha^{0} x_{t-1}+\varepsilon_{x t}, \quad\left(\varepsilon_{y t}, \varepsilon_{x t}\right)^{\prime} \sim(0, \Omega), \quad \Omega=\left(\begin{array}{cc}
\sigma_{y y} & \sigma_{y x} \\
\sigma_{x y} & \sigma_{x x}
\end{array}\right) .
\end{aligned}
$$

Let $\theta=(\beta, \alpha)$ and as before, $\Gamma_{j}=E_{\theta}\left(\varepsilon_{t} \varepsilon_{t-j}^{\prime}\right)=0$ for all $j \neq 0$ with $\Gamma_{0}=\Omega^{0}$. Let $\widehat{\gamma}_{O L S}$ be obtained from least squares regression of $y_{t}$ on $x_{t-1}$ and $z_{t}$, and let $\widehat{\Omega}_{O L S}$ be the estimated covariance matrix of the errors. Since $z_{t}$ is stationary, the estimator $\widehat{\gamma}_{O L S}$ is $\sqrt{T}$ consistent and asymptotically normal uniformly over $\alpha \in(-1+\delta, 1]$. Define the quasi-differenced sequence

$$
e_{y t}=y_{t}-x_{t-1} \beta-z_{t} \widehat{\gamma}_{O L S}, \quad e_{x t}=x_{t}-\alpha x_{t-1}, \quad e_{t}=\left(\begin{array}{c}
e_{y t} \\
e_{x t}
\end{array}\right) \text {. }
$$

Let $\widehat{\Gamma}_{j}(\theta)=\frac{1}{T} \sum_{t=j+1}^{T} e_{t} e_{t-j}^{\prime}$ and define $\bar{g}_{j, Q D}(\theta, \Omega)$ as in the absence of $z_{t}$. Using arguments analogous to Proposition 4 , it can be shown that $\widehat{\theta}_{K, Q D}$ is still $\sqrt{T}$ consistent and asymptotically 
normal. Proposition 4 assumes that the regression error $\varepsilon_{y t}$ is white noise. This is not restrictive as lags of $\Delta y_{t}$ and $\Delta x_{t}$ can be added to $z_{t}$ to control for residual serial correlation.

\section{Non-Linear Models and Minimum Distance Estimation}

So far, the QD framework has been used to estimate linear models, where the model autocovariances are such that $\gamma_{j}(\theta)=0$ for all $\theta$ assumed to be the true value and $j \geq 1$. The analysis also holds if $\gamma_{j}(\theta)$ equals a constant vector other than zero provided that the constant vector is known or can be computed numerically. For example, if $x_{t}$ is an $\operatorname{ARMA}(1,1)$ instead of an $\operatorname{AR}(1), \gamma_{j}(\theta)$ will depend on the parameters of the model. Another example is DSGE models which we now consider.

To fix ideas, consider the simple one sector stochastic growth model presented in Uhlig (1999). Let $Q_{t}, C_{t}, K_{t}, I_{t}$ be output, consumption, capital stock, and investment, respectively. The problem facing the central planner is to maximize expected utility $E_{t-1} \sum_{t=0}^{\infty}(1+\rho)^{-t} \log C_{t}$ subject to the constraints $Q_{t}=K_{t-1}^{\psi} Z_{t}^{1-\psi}=C_{t}+I_{t}$ and $K_{t}=(1-\delta) K_{t-1}+I_{t}$ where $u_{t}=\log Z_{t}$ evolves as

$$
u_{t}=\alpha u_{t-1}+\varepsilon_{t}, \quad \varepsilon_{t} \sim\left(0, \sigma^{2}\right)
$$

Denote the deviation of a variable from its mean by lower case letters. Let $Y_{t}=\left(y_{1 t}, \ldots, y_{N t}\right)^{\prime}$ be the collection of endogenous variables in the model (such as consumption, output, etc.). As shown in Uhlig (1999), this simple model has an analytic solution:

$$
k_{t}=v_{k k} k_{t-1}+v_{k z} u_{t}
$$

where $v_{k k}<1$ does not depend on $\alpha$, but $v_{k z}$ depends on $\alpha$. For each $y_{n t} \in Y_{t}$,

$$
\left(1-v_{k k} L\right)(1-\alpha L) y_{n t}=u_{t}+\vartheta_{n} u_{t-1}
$$

is an $\operatorname{ARMA}(2,1)$ with a moving-average parameter $\vartheta_{n}$ that is a function of the structural parameters. Note that all series in $Y_{t}$ have the same autoregressive dynamics as $k_{t}$. The parameters of the linearized solution are $\beta=\left(v_{k k}, v_{k z}, \vartheta_{1}, \ldots, \vartheta_{N}\right)$. The parameters of the model are $\theta=\left(\psi, \alpha, \sigma^{2}, \rho, \delta\right)$. Let $\Theta$ be a compact set containing possible values of $\theta$.

In analysis of DSGE models, whether the shocks have permanent or transitory effects matter for how a model is to be linearized. For this reasons, researchers typically need to decide whether to difference the data ahead of estimation even though it is understood that the assumption affects the estimates and policy analysis. To date, there does not exist an estimator of DSGE models that 
has classical properties for all values of $\alpha$ within the likelihood framework because the likelihood function is not well defined when the data are non-stationary. ${ }^{6}$

We propose to estimate the parameters of the model without making a priori assumptions about the degree of persistence of the shocks. We use the fact that the features of covariance stationary processes are completely summarized by their second moments. Conveniently, the software DYNARE automatically calculates the covariance structure of the data. Even though the analysis is not likelihood based, priors can still be incorporated using the approach of Chernozhukov and Hong (2003). ${ }^{7}$ The key is to construct the moments $\bar{g}(\theta)$ appropriately.

Two variations of the $\mathrm{QD}$ framework are considered. The first method proceeds as follows. For given $\theta$, let $e_{t}=Y_{t}-\alpha Y_{t-1}$ with $\Gamma_{j}(\theta)=E\left(e_{t} e_{t}^{\prime}\right)$. Define the moment $\omega_{j}(\theta)=\left(\Gamma_{j}(\theta)-\Gamma_{0}(\theta)\right)$ whose sample analog is $\widehat{\omega}_{j}(\theta)=\left(\widehat{\Gamma}_{j}(\theta)-\widehat{\Gamma}_{0}(\theta)\right)$. Note that since $e_{t}(\theta)$ can be serially correlated, $\Gamma_{j}(\theta)$ need not be a null matrix as in the applications considered thus far. Let $\bar{g}_{Q D}(\theta)=\left(\bar{g}_{1, Q D}(\theta)^{\prime}, \ldots, \bar{g}_{K, Q D}(\theta)^{\prime}\right)^{\prime}$ where $\bar{g}_{j, Q D}(\theta)=\operatorname{vec}\left(\widehat{\omega}_{j}(\theta)-\omega_{j}(\theta)\right)$. The QD estimator considered in Gorodnichenko and $\mathrm{Ng}$ (2010) is defined $\mathrm{as}^{8}$

$$
\widehat{\theta}_{K, Q D}=\underset{\theta \in \Theta}{\operatorname{argmin}} \bar{g}_{Q D}(\theta)^{\prime} \bar{g}_{Q D}(\theta) .
$$

As written, $\widehat{\theta}_{K, Q D}$ is an equally weighted estimator. An optimal weighting matrix can be used subject to constraints imposed by stochastic singularity. In the one-shock stochastic growth model considered, the autocovariance at lag one of both output and consumption can both be used to construct an efficient $\widehat{\theta}_{K, Q D}$, but additional autocovariances will not add independent information. In contrast, the use of data for both output and consumption in likelihood estimation would not even be possible.

Another QD-based estimator can be obtained if we entertain the possibility of a reduced form model. Consider a finite order $\mathrm{AR}(\mathrm{p})$ model:

$$
y_{t}=a^{0} y_{t-1}+\sum_{j=1}^{p-1} b_{j}^{0} \Delta y_{t-j}+\varepsilon_{t p}
$$

where $\beta^{0}=\left(a^{0}, b_{1}^{0}, . ., b_{p-1}^{0}\right)=\beta\left(\theta^{0}\right)$ are the true 'reduced-form' parameters that can be computed analytically or numerically. We also need the following:

\footnotetext{
${ }^{6}$ Likelihood estimation is also problematic when there are more variables than shocks, a problem known as stochastic singularity.

${ }^{7}$ For an example of this implementation, see Coibion and Gorodnichenko (2011).

${ }^{8}$ Identification requires $\operatorname{rank} \frac{\partial \bar{g}_{j, Q D}(\theta)}{\partial \theta}=\operatorname{dim} \theta$. Now $\bar{g}_{j}(\theta)$ depends on $\theta$ through the parameters in the solution to expectation equations. Formal identification conditions are given in Komunjer and $\mathrm{Ng}(2011)$.
} 
Assumptions B. (Identification) (i) there is a unique $\theta^{0}$ such that $\beta\left(\theta^{0}\right)=\beta^{0}$; (ii) the function $\beta(\theta)$ is twice continuously differentiable; (iii) $B\left(\theta^{0}\right)=\nabla \beta\left(\theta^{0}\right)$ has full rank $k=\operatorname{dim}(\theta) \leq p$.

The method proceeds as follows. For $y_{n t} \in Y_{t}$, define $e_{n t}(\beta)=y_{n t}-a y_{n t-1}-\sum_{j=1}^{p-1} b_{j} y_{n t-j}$ where $\beta=\left(a, b_{1}, \ldots, b_{p-1}\right)$. Note that $Y_{t}$ is now quasi-differenced using the 'reduced-form' parameter $\beta$ instead of the structural parameter $\alpha$ as in method 1. Once the data are quasi-differenced, estimation proceeds by defining $\omega_{j}(\theta ; p)=\left(\Gamma_{j}(\theta ; p)-\Gamma_{0}(\theta ; p)\right)$ with $\bar{g}_{j, Q D}(\theta ; p)=\widehat{\omega}_{j}(\theta ; p)-\omega_{j}(\theta ; p)$ and sample analog as in $(15)$. Let $\bar{g}_{Q D}(\theta ; p)=\left(\bar{g}_{1, Q D}(\theta ; p)^{\prime}, \ldots, \bar{g}_{K, Q D}(\theta ; p)^{\prime}\right)^{\prime}$. The estimator is

$$
\widehat{\theta}_{K, Q D}=\underset{\theta \in \Theta}{\operatorname{argmin}} \bar{g}_{Q D}(\theta ; p)^{\prime} \bar{g}_{Q D}(\theta ; p) \text {. }
$$

Because $\beta$ is $p$-dimensional, this second estimator also depends on the choice of $p$. Since $e_{n t}$ is not necessarily exactly white noise, $\Gamma_{j}(\theta)$ will not be zero. However, its autocovariances can be computed for any given $\theta$.

We have presented two uses of the QD framework that can yield estimators that are robust to non-stationary exogenous variables in DSGE models. The data-dependent transformations allow us to construct moments that are uniformly bounded. Applying the central limit theorem to the sample moments yields estimators with classical properties.

\section{Simulations}

We consider the finite sample properties of OLS, FQD with $\gamma_{0}$ fixed at the true $\sigma_{0}^{2}$, and QD. Even though the FQD estimator is infeasible in practice, it is a useful benchmark. The simulations are based on 2,000 replications. We use the standard Newey-West plug-in estimator for the variance of the moments. As starting values, we use 0.9 times the true values of the parameters. The QD estimator requires evaluation of the model implied autocovariances $\Gamma_{j}(\theta)$. This is straightforward once a model is cast in a state space. For example, the system

$$
\left[\begin{array}{l}
y_{t} \\
x_{t}
\end{array}\right]=\left[\begin{array}{ll}
0 & \beta \\
0 & \alpha
\end{array}\right]\left[\begin{array}{l}
y_{t-1} \\
x_{t-1}
\end{array}\right]+\left[\begin{array}{ll}
1 & 0 \\
0 & 1
\end{array}\right]\left[\begin{array}{l}
\varepsilon_{y t} \\
\varepsilon_{x t}
\end{array}\right]
$$

in quasi-differenced form is

$$
\left[\begin{array}{l}
e_{y t}(\theta) \\
e_{x t}(\theta)
\end{array}\right]=\left[\begin{array}{ll}
0 & 0 \\
0 & 0
\end{array}\right]\left[\begin{array}{l}
e_{y t-1}(\theta) \\
e_{x t-1}(\theta)
\end{array}\right]+\left[\begin{array}{ll}
1 & 0 \\
0 & 1
\end{array}\right]\left[\begin{array}{l}
\varepsilon_{y t} \\
\varepsilon_{x t}
\end{array}\right] .
$$


More generally, every ARMA model has a state-space representation from which a state-space model for the quasi-differenced data can be expressed as

$$
w_{t}=D_{0} w_{t-1}+D_{1} \varepsilon_{t}
$$

where $w_{t}$ includes $e_{t}(\theta)$ (and possibly its lags), and $\varepsilon_{t}$ is the set of exogenous white noise shocks with variance $\Omega_{\varepsilon}$. The variance matrix $\Omega_{w}(0)=E\left(w_{t} w_{t}^{\prime}\right)$ can be computed by iterating the equation

$$
\Omega_{w}^{(i)}(0)=D_{0} \Omega_{w}^{(i-1)}(0) D_{0}^{\prime}+D_{1} \Omega_{\varepsilon} D_{1}^{\prime}
$$

until convergence. The autocovariance matrices can then be computed as $\Omega_{w}(j)=D_{0}^{j} \Omega_{w}(0)$. Now $\Gamma_{j}(\theta)$ are submatrices of $\Omega_{w}(j)$. If we are only interested in computing the moments $w_{t}^{d} \subset w_{t}$, we iterate equation (18) until the block that corresponds to $w_{t}^{d}$ converges, i.e. $\left\|\Omega_{w^{d}}^{(i)}(0)-\Omega_{w^{d}}^{(i-1)}(0)\right\|<c$.

Data are generated from the $\operatorname{AR}(2)$ model:

$$
\left(1-\lambda_{1}^{0} L\right)\left(1-\lambda_{2}^{0} L\right) y_{t}=\varepsilon_{t}, \quad \varepsilon_{t} \sim N(0,1)
$$

The process can be written as

$$
y_{t}=\alpha^{0} y_{t-1}+b^{0} \Delta y_{t-1}+\varepsilon_{t}
$$

The parameter of interest is $\alpha^{0}=\lambda_{1}^{0}+\lambda_{2}^{0}-\lambda_{1}^{0} \lambda_{2}^{0}$ with $b^{0}=\lambda_{1}^{0} \lambda_{2}^{0}$. The OLS estimate of $\alpha$ has a non-standard distribution when the roots are unity, in which case $\alpha^{0}=1$.

We estimate an $\operatorname{AR}(2)$ model when $\lambda_{2}^{0}=0$ (Table 1). Demeaned data are used to compute the sample autocovariances in the intercept case, and linearly de-trended data are used in the linear trend case. We report the mean of the QD, FQD, and OLS estimates when $T=200$ and 500, the $J$ test for over-identifying restrictions, along with the finite sample power for one-sided $t$ tests evaluated at $\alpha=\alpha^{0}-0.05$.

Table 1 shows that all three estimators are precise when $\alpha^{0} \leq 0.8$. The $t$-statistic for the null hypothesis that $\alpha=\alpha^{0}$ for all three estimators has rejection rates close to the nominal size of 0.05 when $\alpha^{0} \leq 0.8$. The picture is, however, very different at larger values of $\alpha^{0}$. The FQD has slightly smaller bias but is much less efficient. While OLS has the largest bias, its root-mean-squared error (RMSE) is much smaller than the FQD. The QD is neither the most accurate nor the most efficient, but has RMSE closer to OLS and much smaller than the FQD, in support of Proposition 2.

Efficiency of OLS comes at the cost of size distortion, however. At $T=200$, the OLS-based $t$ statistic has a rejection rate of 0.473 when $\alpha^{0}=1$ and 0.15 when $\alpha^{0}=0.95$, much larger than 
the nominal size of 0.05 . Even at $T=500$, the rejection rates are 0.462 and 0.127 , well above the nominal rate of 5\%. The rejection rates for the FQD and QD are 0.107 and 0.08 when $T=200$, and are 0.066 and 0.076 when $T=500$, much closer to the nominal size of 0.05 . The QD has accurate rejection rates that are always around 0.05 for all values of $\alpha^{0}$, but it has less power than OLS. Figure 1 plots the distribution of t-statistics for QD at $T=200$ and $T=500$. The normal approximation to the finite sample distribution is good.

We report results for over-parameterized $\mathrm{AR}(\mathrm{p})$ models in Table 2. In particular, we estimate an $\operatorname{AR}(3)$ when the true model is an $\operatorname{AR}(2)$. We fix $\lambda_{2}^{0}$ to 0.2 and set $\lambda_{1}^{0}$ such that the sum of the $\operatorname{AR}(2)$ parameters is the same as in Table 1. Statistics are reported for $\widehat{\alpha}$. The results are similar to Table 1: the FQD is inefficient; the $t$ statistic associated with it has good size but lower power. The OLS has large size distortion when $\alpha^{0}$ is close to one, but the estimates are tightly estimated. The QD strikes a balance between the two, and the $t$ statistic has very similar size property for all values of $\left|\alpha^{0}(1)\right| \leq 1$ even when $T=200$ in the intercept case. In the linear time trend case, the uniform size property can be achieved with $T=500$. Importantly, the QD achieves a more accurate size when $\alpha^{0}$ is close to unity without sacrificing too much power outside of the persistent range. For example, when $\alpha^{0}$ is 0.5 , the power of OLS and QD are quite similar. The reason is that when $\alpha^{0}$ is far from the unit circle, OLS is $\sqrt{T}$ consistent, but so is FQD and QD. Power is also fairly similar for both the intercept only and the linear trend model.

Table 3 presents results for predictive regressions. Data for the predictive regressions are generated as in (14a) and (14b). We let $\beta^{0}=1, \sigma_{y y}^{0}=1, \sigma_{x x}^{0}=1, \sigma_{y x}^{0}=0.5$. The results are generally similar to the $\mathrm{AR}(\mathrm{p})$ case. The QD is slightly less efficient than OLS when $\alpha^{0}$ is close to one but no less efficient when $\alpha^{0}$ is far from the unit circle. This loss of efficiency arises from the fact that at $\alpha^{0}=1$, OLS is $T$ consistent but the QD is only $\sqrt{T}$ consistent. The benefit to this small efficiency loss is robustness. As one can see, the size of the $t$ test for $H_{0}: \beta=\beta^{0}$ is fairly constant around the nominal size of 0.05 , but the size of the test based on OLS varies with $\alpha^{0}$. Furthermore, as $\sigma_{y x}^{0}$ rises, the size of the t-test based on OLS becomes increasingly distorted while QD continues to have sizes close to nominal.

Finally, we consider the stochastic growth model presented in Section 5. The data are generated as follows. We fix the true value of capital intensity $\psi$ to 0.25 and $\sigma^{2}$ to 1 and consider five values of $\alpha: 1,0.98,0.95,0.9,0.8$. We use data on consumption to estimate $\psi, \alpha$ and $\sigma^{2}$ using the second method discussed in Section 5. This consists of solving for $\psi, \alpha$ and $\sigma$ from the six autocovariances of $e_{t}(\beta)$, where $\beta$ are the parameters of an $\operatorname{AR}(3)$ model. For the sake of comparison, we also use 
the Kalman filter to obtain the maximum likelihood estimates. The results are reported in Table 4. Evidently, the QD estimates of $\psi$ are close to the true value of 0.25 , and the size of the $t$-test is close to the nominal value for all values of $\alpha$. In contrast, the MLE estimates are biased when $\alpha$ is close to one, and the $t$ test for the null hypothesis that $\psi=0.25$ is severely distorted.

\section{Relation to other $\sqrt{T}$-Consistent Linear Estimators}

As discussed in Section 2, one of the problems with the OLS estimator when a unit root is present is that the moment condition at the true value $\sqrt{T} \bar{g}\left(\alpha^{0}\right)=\frac{1}{\sqrt{T}} \sum_{t=1}^{T} y_{t-1} \varepsilon_{t}$ does not satisfy a central limit theorem. Although $y_{t-1}$ is orthogonal to $\varepsilon_{t}$, the persistence of $y_{t-1}$ requires a stronger normalization and standard distribution theory cannot be used. The thrust of QD estimator is to use moment conditions that satisfy a central limit theorem uniformly over values of $\alpha^{0}$. The approach can be used to estimate a broad range of models. However, for the simple AR(1) model, the ideas underlying the QD estimation can be used to construct a two-step linear estimator. We now show how this can be done and then relate this approximate QD estimator to other known linear estimators of the $\mathrm{AR}(1)$ model with classical properties in the local-to-unity framework.

For the AR(1) model, the QD moment condition (3) replaces $y_{t-1}$ with $\varepsilon_{t-j}$. As seen from equation (4), the central limit theorem holds whether or not there is a unit root present. But the moment condition can be understood in an instrumental variable setup because $\varepsilon_{t-j}$ is uncorrelated with $\varepsilon_{t}$ and is hence a valid instrument. The only problem is that $\varepsilon_{t-j}$ is not observed. But $\widehat{\alpha}_{O L S}$ is consistent for all $\left|\alpha^{0}\right| \leq 1$. Thus, let $\widetilde{e}_{t-1}=y_{t}-\widehat{\alpha}_{O L S} y_{t-1}$, noting that generated instruments do not require a correction for the standard errors like generated regressors do. We can now define a (hybrid differencing) HD estimator using the following moment condition: ${ }^{9}$

$$
\bar{g}_{H D}\left(\widehat{\alpha}_{H D}\right)=\frac{1}{T} \sum_{t=k+1}^{T} \widetilde{e}_{t-k}\left(y_{t}-\widehat{\alpha}_{H D} y_{t-1}\right)=0 .
$$

This leads to the estimator

$$
\widehat{\alpha}_{H D}=\frac{\sum_{t=k}^{T} y_{t} \widetilde{e}_{t-k}}{\sum_{t=k}^{T} y_{t-1} \widetilde{e}_{t-k}}=\alpha^{0}+\frac{\sum_{t=k}^{T} e_{t} \widetilde{e}_{t-k}}{\sum_{t=k}^{T} y_{t-1} \widetilde{e}_{t-k}} .
$$

We refer to $\widehat{\alpha}^{H D}$ as a hybrid estimator because it is based on the covariance between the quasidifference of $y_{t}$ and a stationary random variable. Notice that the HD and QD use the same moment

\footnotetext{
${ }^{9}$ Laroque and Salanie (1997) used two OLS regressions in stationary variables to obtain a $\sqrt{T}$-consistent estimate of the co-integrating vector.
} 
condition. What distinguishes the HD from the QD is that the objective function of the HD is now linear in $\alpha$. Consistency of $\widehat{\alpha}_{H D}$ follows from the fact that $\frac{1}{T} \sum_{t=1}^{T} e_{t}\left(\alpha^{0}\right) \widetilde{e}_{t-k} \stackrel{p}{\longrightarrow} E \varepsilon_{t} \varepsilon_{t-k}=0$. It is straightforward to show that in the local-to-unity framework,

$$
\sqrt{T}\left(\widehat{\alpha}_{H D}-\alpha^{0}\right) \Rightarrow 2\left(1+J_{c}(1)^{2}\right)^{-1} N(0,1) .
$$

Once the HD is understood as an instrumental estimator, other possibilities arise. Instead of $\widetilde{e}_{t-1}$, we can use any stationary series uncorrelated with the error term. ${ }^{10}$ For example, using $\Delta y_{t-1}$ would by-pass the need for a preliminary least squares estimation. The first differencing (FD) estimator is:

$$
\widehat{\alpha}_{F D}=\frac{\sum_{t=2}^{T} \Delta y_{t-1} y_{t}}{\sum_{t=2}^{T} \Delta y_{t-1} y_{t-1}} .
$$

The FD is a special case of estimators analyzed in So and Shin (1999). These authors used the sign of $y_{t-1}$ as instrument $x_{t}$ to construct

$$
\widehat{\alpha}_{S S}=\frac{\sum_{t=2}^{T} x_{t} y_{t}}{\sum_{t=1}^{T} x_{t} y_{t-1}} .
$$

Another estimator with classical properties in the local-to-unity framework is that of Phillips and Han (2008). The PH estimator, defined as

$$
\widehat{\alpha}_{P H}=\frac{\sum_{t=2}^{T} \Delta y_{t-1}\left(2 \Delta y_{t}+\Delta y_{t-1}\right)}{\sum_{t=2}^{T}\left(\Delta y_{t-1}\right)^{2}},
$$

has the property that $\sqrt{T}\left(\widehat{\alpha}_{P H}-\alpha^{0}\right) \Rightarrow N\left(0,2\left(1+\alpha^{0}\right)\right)$ for all $\alpha^{0} \in(-1,1]$. As shown in the Appendix, the FD estimator is asymptotically equivalent to the $\mathrm{PH}$ estimator in the stationary case when $\alpha^{0}$ is far from unit circle. That is, for the $\operatorname{AR}(1)$ model, $\widehat{\alpha}_{P H}=\widehat{\alpha}_{F D}+O_{p}(1 / T)$ under stationary classical asymptotics. However, these two estimators differ in the local-to-unity setting. While $\sqrt{T}\left(\widehat{\alpha}_{F D}-\alpha^{0}\right) \Rightarrow 2\left(1+W(1)^{2}\right)^{-1} N(0,1)$ when $\alpha^{0}=1, \sqrt{T}\left(\widehat{\alpha}_{P H}-\alpha^{0}\right) \Rightarrow N(0,4)$. The FD is thus more efficient at $\alpha^{0}=1$. Simulations presented in Table 5 show that the QD dominates the FD and $\mathrm{PH}$, but is comparable to the HD. The simulations also support the theoretical predictions that the FD, HD, and QD are all asymptotically normal and $\sqrt{T}$ consistent.

No estimator is perfect and QD estimation has its drawbacks. As mentioned in the introduction, the price we pay for asymptotic normality is that $\widehat{\alpha}_{Q D}$ converges at a rate of $\sqrt{T}$ instead of $T$ when

\footnotetext{
${ }^{10}$ As suggested by a referee, $E\left(e_{t}\left(e_{t-j}-e_{t-k}\right)\right)=0,1 \leq j<k$ is also a valid moment condition.
} 
there is a unit root. Han et al. (2011) aggregate $L$ stationary moment conditions and showed that by suitable choice of $L$, uniform asymptotic normality can be achieved at a rate faster than $\sqrt{T}$. Extension of their result outside of the $\mathrm{AR}(\mathrm{p})$ model is, however, not straightforward. In contrast, the QD framework is broadly applicable.

\section{Concluding Comments}

In this paper, we use a quasi-differencing framework to obtain estimators with classical properties even when the underlying data are highly persistent. Quasi-differencing can render non-stationary processes stationary so that classical limit theorems can be applied. However, the QD estimator is $\sqrt{T}$ consistent rather than super-consistent in the local-to-unity framework. In exchange for this slower convergence is generality, as QD estimation can be used in a broad range of linear and non-linear models. However, there are several issues that remain to be solved. The first is allowing $J$ to be data dependent and increase with the sample size. The second is to allow for conditional heteroscedasticity. Third, simulations suggest that the QD works well even when forcing process is mildly explosive. Relaxing the assumption that the largest autoregressive root is inside the unit disk may well be useful for practitioners. These issues are left for future investigation. 


\section{References}

Abowd, J. M. and Card, D. 1989, On the Covariance Structure of Earnings and Hours Changes, Econometrica 57(2), 411-445.

Altonji, J. G. and Segal, L. M. 1996, Small-Sample Bias in GMM Estimation of Covariance Structures, Journal of Business and Economic Statistics 14(3), 353-366.

Canjels, E. and Watson, M. W. 1997, Estimating Deterministic Trends in the Presence of Serially Correlated Errors, Review of Economics and Statistics 79(2), 184-200.

Chernozhukov, V. and Hong, H. 2003, An MCMC Approach to Classical Estimation, Journal of Econometrics 115(2), 293-346.

Coibion, O. and Gorodnichenko, Y. 2011, Strategic Interaction Among Heterogeneous Price Setters in Estimated DSGE Model, Review of Economics and Statistics 93(3), 920-940.

Gorodnichenko, Y. and Ng, S. 2010, Estimaton of DSGE Models When the Data are Persistent, Journal of Monetary Economics 57(3), 325-340.

Han, C. and Kim, B. 2011, A GMM Interpretation of the Paradox in the Inverse Probability Weighting Estimation of the Average Treatment Effect on the Treated, Economics Letters 110, 163-165.

Han, C., Phillips, P. C. B. and Sul, D. 2011, Uniform Asymptotic Normality in Stationary and Unit Root Autoregression, Econometric Theory, forthcoming.

Jansson, M. and Moreira, M. J. 2006, Optimal Inference In Regession Models with Nearly Integrated Regressors, Econometrica 74, 681-714.

Komunjer, I. and Ng, S. 2011, Dynamic Identification of DSGE models, Econometrica, forthcoming.

Laroque, G. and Salanie, B. 1997, Normal Estimators for Cointegrating Relationships, Economics Letters 55(2), 185-189.

Mikusheva, A. 2007a, Uniform Inference in Autoregressive Models, Econometrica 75(5), 1411-1452.

Mikusheva, A. 2007b, Uniform Inference in Autoregressive Models, Econometrica 75(5), Supplementary Material.

Mikusheva, A. 2011, One Dimensional Inference in Autogressive Models with the Potential Presence of a Unit Root, Econometrica. forthcoming.

Newey, W. K. and McFadden, D. 1994, Large Sample Estimation and Hypothesis Testing, Handbook of Econometrics, Vol. 4,Chapter 36, North Holland.

Pesavento, E. and Rossi, B. 2006, Small-sample Confidence Interevals for Multivariate Impulse Response Functions at Long Horizons, Journal of Applied Econometrics 21(8), 1135-1155.

Phillips, P. C. B. and Han, C. 2008, Gaussian Inference in AR(1) Time Series With or Without a Unit Root, Econometric Theory 24, 631-650.

Phillips, P. C. B. and Lee, C. C. 1996, Efficiency Gains from Quasi-differencing under Nonstationarity, in P. Robinson and M. Rosenblatt (eds), Athens Conference on Applied Probability and Time Series: Volume II Time Series Analysis in Honor of E.J. Hannan.

Phillips, P. C. B. and Solo, V. 1992, Asymptotics for Linear Processes, Annals of Statistics 20(2), 971-1001. 
Phillips, P. C. B. and Xiao, Z. 1998, A Primer on Unit Root Testing, Journal of Economic Surveys 12(5), 423-469.

Pierce, D. A. 1982, The Asymptotic Effect of Substituting Estimators for Parameters in Certain Types of Statistics, Annals of Statistics 10(2), 475-478.

Prokhorov, A. and Schmidt, P. 2009, GMM Redundancy Results for General Missing Data Problem, Journal of Econometrics 151(1), 47-55.

So, B. S. and Shin, D. W. 1999, Cauchy Estimators for Autoregressive Processes with Applications to Unit Root Tests and Confidence Intervals, Econometric Theory 15(2), 165-176.

Uhlig, H. 1999, A Toolkit for Analyzing Nonlinear Dynamic Stochastic Models Easily, in R. Marimon and A. Scott (eds), Computational Methods for the Study of Dynamic Economies, Oxford University Press, pp. 30-61. 


\section{Appendix A Proofs}

The proofs proceed with the assumption that the weighting matrix $W_{T}$ is an identity matrix.

Proof of Proposition 1 (i): $\quad$ First, consider the problem of matching the $j$-th autocovariance. That is, $Q_{j}(\alpha)=\left(\widehat{\gamma}_{j}(\alpha)-\gamma_{j}(\alpha)\right)^{2}$, and $\widehat{\alpha}_{j}=\arg \min _{\alpha} Q_{j}(\alpha)$. Under the assumption that $\alpha$ is the true value, $\gamma_{0}(\alpha)=\sigma^{2}$, and $\gamma_{j}(\alpha)=0$ for all $j>0$. Note that

$\widehat{\gamma}_{j}(\alpha)-\gamma_{j}(\alpha)=\frac{1}{T} \sum_{t=j+1}^{T} \varepsilon_{t} \varepsilon_{t-j}-\gamma_{j}(\alpha)-\frac{\alpha-\alpha^{0}}{T} \sum_{t=j+1}^{T}\left[\varepsilon_{t} y_{t-j-1}+\varepsilon_{t-j} y_{t-1}\right]+\frac{\left(\alpha-\alpha^{0}\right)^{2}}{T} \sum_{t=j+1}^{T} y_{t-1} y_{t-j-1}$.

As a result, the NQD objective function is the fourth-order polynomial:

$$
Q_{j}(\alpha)=Q_{j}^{(0)}+\left(\alpha-\alpha^{0}\right) Q_{j}^{(1)}+\left(\alpha-\alpha^{0}\right)^{2} Q_{j}^{(2)}+\left(\alpha-\alpha^{0}\right)^{3} Q_{j}^{(3)}+\left(\alpha-\alpha^{0}\right)^{4} Q_{j}^{(4)} .
$$

In the local-to-unity framework with $\alpha_{0}=1+c / T$, the following results hold as $T \rightarrow \infty$ :

$$
\begin{aligned}
\frac{1}{T} \sum \varepsilon_{t-j} y_{t-1} & \Rightarrow \sigma^{2}+\sigma^{2} \int_{0}^{1} J_{c}(s) d W(s), \\
\frac{1}{T} \sum \varepsilon_{t} y_{t-1-j} & \Rightarrow \sigma^{2} \int_{0}^{1} J_{c}(s) d W(s), \\
\frac{1}{T^{2}} \sum y_{t-1} y_{t-j-1} & \Rightarrow \sigma^{2} \int_{0}^{1} J_{c}^{2}(s) d s .
\end{aligned}
$$

It follows from equations (4) and (A.2) - (A.4) that

$$
\begin{aligned}
T^{1 / 2} Q_{j}^{(1)}= & -2 \sqrt{T}\left(\frac{1}{T} \sum_{t=j+1}^{T} \varepsilon_{t} \varepsilon_{t-j}-\gamma_{j}(\alpha)\right) \frac{1}{T} \sum_{t=j+1}^{T}\left[\varepsilon_{t} y_{t-j-1}+\varepsilon_{t-j} y_{t-1}\right] \\
\Rightarrow & -2 \xi_{j}\left(\sigma^{2}+2 \sigma^{2} \int_{0}^{1} J_{c}(s) d W(s)\right) ; \\
T^{-1 / 2} Q_{j}^{(2)} & =2 \sqrt{T}\left(\frac{1}{T} \sum_{t=j+1}^{T} \varepsilon_{t} \varepsilon_{t-j}-\gamma_{j}(\alpha)\right)\left(\frac{1}{T^{2}} \sum_{t=j+1}^{T} y_{t-1} y_{t-j-1}\right) \\
& +\frac{1}{\sqrt{T}}\left(\frac{1}{T} \sum_{t=j+1}^{T}\left[\varepsilon_{t} y_{t-j-1}+\varepsilon_{t-j} y_{t-1}\right]\right)^{2} \Rightarrow 2 \xi_{j} \sigma^{2} \int_{0}^{1} J_{c}^{2}(s) d s \\
T^{-1} Q_{j}^{(3)}= & -2\left(\frac{1}{T^{2}} \sum_{t=j+1}^{T} y_{t-1} y_{t-j-1}\right)\left(\frac{1}{T} \sum_{t=j+1}^{T}\left[\varepsilon_{t} y_{t-j-1}+\varepsilon_{t-j} y_{t-1}\right]\right) \\
& \Rightarrow-2 \sigma^{2} \int_{0}^{1} J_{c}^{2}(s) d s\left(\sigma^{2}+2 \sigma^{2} \int_{0}^{1} J_{c}(s) d W(s)\right) ;
\end{aligned}
$$




$$
T^{-2} Q_{j}^{(4)}=\left(\frac{1}{T^{2}} \sum_{1}^{T} y_{t-1} y_{t-2}\right)^{2} \Rightarrow\left(\sigma^{2} \int_{0}^{1} J_{c}^{2}(s) d s\right)^{2}>0
$$

where $\sqrt{T}\left(\frac{1}{T} \sum \varepsilon_{t} \varepsilon_{t-j}-\gamma_{j}\right) \Rightarrow \xi_{j}=N\left(0, \sigma^{4}\right)$. To summarize:

$$
Q_{j}^{(1)}=O_{p}\left(T^{-1 / 2}\right), \quad Q_{j}^{(2)}=O_{p}\left(T^{1 / 2}\right), \quad Q_{j}^{(3)}=O_{p}\left(T^{1}\right), \quad Q_{j}^{(4)}=O_{p}\left(T^{2}\right) .
$$

It follows that

$$
\frac{Q_{j}(\alpha)-Q_{j}^{(0)}}{T^{2}} \Rightarrow\left(\sigma^{2} \int_{0}^{1} J_{c}^{2}(s) d s\right)^{2}\left(\alpha-\alpha^{0}\right)^{4}
$$

uniformly over a bounded parameter space for $\alpha$. As a result, $\widehat{\alpha}_{j}$ is a consistent estimate of $\alpha^{0}$.

To study the large sample properties of $\widehat{\alpha}_{j}$, consider the first order condition:

$$
Q_{j}^{(1)}+2\left(\widehat{\alpha}_{j}-\alpha^{0}\right) Q_{j}^{(2)}+3\left(\widehat{\alpha}_{j}-\alpha^{0}\right)^{2} Q_{j}^{(3)}+4\left(\widehat{\alpha}_{j}-\alpha^{0}\right)^{3} Q_{j}^{(4)}=0 .
$$

This is a cubic equation of the form $a x^{3}+b x^{2}+c x+d=0$, where $x$ stands for $\left(\widehat{\alpha}_{j}-\alpha_{0}\right)$ with the obvious correspondence between the coefficients. The cubic equation may have one or three real roots depending on the sign of the determinant:

$$
\Delta=18 a b c d-4 b^{3} d+b^{2} c^{2}-4 a c^{3}-27 a^{2} d^{2} .
$$

Given the orders established in (A.5), it can be shown that

$$
T^{-7 / 2} \Delta \Rightarrow-32 \sigma^{10}\left(\int_{0}^{1} J_{c}^{2}(s) d s\right)^{5} \xi_{j}^{3}
$$

The sign of the determinant $\Delta$ is asymptotically defined by the sign of $\xi_{j}$. When the sign of $\Delta$ is negative, there is a unique real root to equation (A.6); otherwise, there are three real roots. However, in the case of three real roots, the middle one corresponds to the local maxima, while the other two roots are the local minima of (A.1).

The next step is to work out the formulas for the roots and to check their rates of convergence toward zero. For example, when there is only one real root, the formula is:

$$
x_{1}=-\frac{1}{3 a}\left(b+\sqrt[3]{b^{3}-\frac{9}{2} a b c+\frac{27}{2} a^{2} d+\frac{1}{2} \sqrt{-27 a^{2} \Delta}}+\sqrt[3]{b^{3}-\frac{9}{2} a b c+\frac{27}{2} a^{2} d-\frac{1}{2} \sqrt{-27 a^{2} \Delta}}\right) .
$$

Using the asymptotic orders of the terms in (A.5) and after tedious algebra, we can deduce that $T x_{1}=O_{p}(1)$ (that is $\left.T\left(\widehat{\alpha}_{j}-\alpha^{0}\right)=O_{p}(1)\right)$. Similarly, using the explicit formula for cubic roots and denoting the two non-central roots by $x_{2}$ and $x_{3}$, we can deduce that when $\Delta>0, T^{3 / 4} x_{2}=O_{p}(1)$ and $T^{3 / 4} x_{3}=O_{p}(1)$ (that is, $T^{3 / 4}\left(\widehat{\alpha}_{j}-\alpha^{0}\right)=O_{p}(1)$ in this case).

To find the asymptotic distribution of these roots, we start with the case of one root. Because $T\left(\widehat{\alpha}_{j}-\right.$ $\left.\alpha^{0}\right)=O_{p}(1)$, some terms in equation (A.6) are asymptotically negligible. Thus, asymptotically we have $Q_{j}^{(1)}+2\left(\widehat{\alpha}_{j}-\alpha^{0}\right) Q_{j}^{(2)}=0$. Equivalently,

$$
T\left(\widehat{\alpha}_{j}-\alpha^{0}\right)=-T \frac{Q_{j}^{(1)}}{2 Q_{j}^{(2)}}+o_{p}(1) \Rightarrow \frac{\sigma^{2}+2 \sigma^{2} \int_{0}^{1} J_{c}(s) d w(s)}{2 \sigma^{2} \int_{0}^{1} J_{c}^{2}(s) d s} .
$$


Similarly, for the case of two local maxima $T^{3 / 4}\left(\widehat{\alpha}_{j}-\alpha^{0}\right)=O_{p}(1)$ and asymptotically $2\left(\widehat{\alpha}_{j}-\right.$ $\left.\alpha^{0}\right) Q_{j}^{(2)}+4\left(\widehat{\alpha}_{j}-\alpha^{0}\right)^{3} Q_{j}^{(4)}=0$. Equivalently,

$$
T^{3 / 2}\left(\widehat{\alpha}_{j}-\alpha^{0}\right)^{2}=-T^{3 / 2} \frac{Q_{j}^{(2)}}{2 Q_{j}^{(4)}} \Rightarrow \frac{-\xi_{j}}{\sigma^{2} \int_{0}^{1} J_{c}^{2}(s) d s} .
$$

The equation has a solution only when $\xi_{j}<0$. As shown above, this is the condition for the cubic equation to have three roots. Thus, we proved that equation (6) holds for $\widehat{\alpha}_{j}$ with $K=1$. Analogous arguments hold in the general case $\widehat{\alpha}_{K, N Q D}=\arg \min _{\alpha} \sum_{j=1}^{K} Q_{j}(\alpha)$ with $\xi_{j}$ replaced by $\sum_{j=1}^{K} \xi_{j} / K \sim N\left(0, \sigma^{4} / K\right)$. This also shows that in $\operatorname{AR}(1)$ case, matching more than one autocovariance leads to increase in efficiency.

Proposition 1 (ii) and Proposition 2 are special cases of Proposition 3. Observe that

$$
\bar{g}_{j, F Q D}(\beta)=A_{j, F Q D}+\left(\beta^{0}-\beta\right)^{\prime} B_{j, F Q D}+\left(\beta^{0}-\beta\right)^{\prime} C_{j, F Q D}\left(\beta^{0}-\beta\right),
$$

where $A_{j, F Q D}, B_{j, F Q D}$ and $C_{j, F Q D}$ are defined in equations (11)-(13). The following three lemmas will be used to prove Proposition 3.

Lemma A-1 (Uniform Law of Large Numbers) Let $\varepsilon_{t}=\left(\varepsilon_{t, 1}, \varepsilon_{t, 2}\right)^{\prime}$ be martingale-difference sequence with $\Omega=E\left(\varepsilon_{t} \varepsilon_{t}^{\prime} \mid \mathcal{F}_{t-1}\right)$ and finite fourth moments, $\Omega=\left(\sigma_{i, j}\right)$ and $\eta_{t, i}=\sum_{j=0}^{\infty} c_{j}^{i} \varepsilon_{t-j, i}$ for $i=1,2$. Uniformly over the set of all sequences $c_{j}^{i}$ satisfying $\sum_{j=0}^{\infty}\left|c_{j}^{i}\right|<C$ for some constant $C$,

$$
\frac{1}{T} \sum_{t=1}^{T} \eta_{t, 1} \eta_{t, 2} \rightarrow^{p} E\left[\eta_{t, 1} \eta_{t, 2}\right]
$$

Proof of Lemma A-1 Notice first that

$$
\gamma_{j}^{i_{1}, i_{2}}=\operatorname{cov}\left(\eta_{t, i_{1}}, \eta_{t+j, i_{2}}\right)=\sigma_{i_{1}, i_{2}} \sum_{n=0}^{\infty} c_{n+j}^{i_{1}} c_{n}^{i_{2}}
$$

and for any $i_{1}$ and $i_{2}, \sum_{j=0}^{\infty}\left|\gamma_{j}^{i_{1}, i_{2}}\right|<\|\Omega\| C^{2}$. Furthermore,

$$
E\left[\eta_{t, 1} \eta_{t, 2} \eta_{, t+j, 1} \eta_{t+j, 2}\right]=\left(\gamma_{0}^{1,2}\right)^{2}+\gamma_{j}^{1,2} \gamma_{j}^{2,1}+\gamma_{j}^{1,1} \gamma_{j}^{2,2}+E\left(\varepsilon_{1, t}^{2} \varepsilon_{2, t}^{2}\right) \sum_{n=0}^{\infty} c_{n}^{1} c_{n+j}^{1} c_{n}^{2} c_{n+j}^{2} .
$$

As a result,

$$
\operatorname{cov}\left(\eta_{t, 1} \eta_{t, 2}, \eta_{t+j, 1} \eta_{t+j, 2}\right)=\gamma_{j}^{1,2} \gamma_{j}^{2,1}+\gamma_{j}^{1,1} \gamma_{j}^{2,2}+E\left(\varepsilon_{1, t}^{2} \varepsilon_{2, t}^{2}\right) \sum_{n=0}^{\infty} c_{n}^{1} c_{n+j}^{1} c_{n}^{2} c_{n+j}^{2}
$$

and

$$
\sum_{j=1}^{\infty} \operatorname{cov}\left(\left(\eta_{t, 1} \eta_{t, 2}\right),\left(\eta_{t+j, 1} \eta_{t+j, 2}\right)\right) \leq 2\|\Omega\|^{2} C^{4}+E\left(\varepsilon_{t, 1}^{2} \varepsilon_{t, 2}^{2}\right)\left(\sum_{n=0}^{\infty}\left|c_{n}^{1} c_{n}^{2}\right|\right)^{2} \leq\left(2\|\Omega\|^{2}+E\left(\varepsilon_{t, 1}^{2} \varepsilon_{t, 2}^{2}\right)\right) C^{4}
$$

Chebyshev's inequality implies the statement of the Lemma. 
Lemma A-2 The following three statements hold asymptotically uniformly over $\mathfrak{R}_{\delta}$ and uniformly over $1 \leq j \leq K$

(a) $\sqrt{T}\left(A_{1, F Q D}, \ldots, A_{K, F Q D}\right)^{\prime} \Rightarrow\left(\xi_{1}-\xi_{0}, \ldots, \xi_{K}-\xi_{0}\right)$, where $\left(\xi_{0}, \xi_{1}, \ldots, \xi_{K}\right)^{\prime}$ is a normally distributed random vector with mean zero and diagonal covariance matrix, $E \xi_{0}^{2}=\mu_{4}, E \xi_{j}^{2}=\sigma^{4}$ for all $1 \leq j \leq K$;

(b) $B_{j, F Q D} \rightarrow^{p} a_{j}=E\left[\left(X_{t+j}+X_{t-j}-2 X_{t}\right) \varepsilon_{t}\right]$;

(c) $C_{j, F Q D}=O_{p}(1)$.

Proof of Lemma A-2: Part (a) follows from applying the Central Limit Theorem (4) to the sums of $\varepsilon_{t}^{2}-\sigma^{2}$ and $\varepsilon_{t} \varepsilon_{t-j}$ for $1 \leq j \leq K$. For (b) we need to show that the Uniform Law of Large Numbers holds for

$B_{j, F Q D}=\frac{1}{T} \sum_{t=p+j+1}^{T}\left(X_{t} \varepsilon_{t-j}+X_{t-j} \varepsilon_{t}-2 X_{t} \varepsilon_{t}\right)=\frac{1}{T} \sum_{t=p+j+1}^{T-j}\left(X_{t+j}+X_{t-j}-2 X_{t}\right) \varepsilon_{t}+O_{p}(1 / \sqrt{T})$,

where the $O_{p}(1 / \sqrt{T})$ term appears due to the change of limits of summation by a finite number of summands. To apply Lemma A-1, we need to show that the process $X_{t+j}+X_{t-j}-2 X_{t}$ has absolutely summable MA coefficients. From Lemma S8 in the web Appendix of Mikusheva (2007b), the process $Z_{t}=X_{t}-X_{t-1}$ has absolutely summable MA coefficients uniformly over $\mathfrak{R}_{\delta}$. Now

$$
X_{t+j}+X_{t-j}-2 X_{t}=\sum_{k=1}^{j} Z_{t+k}-\sum_{k=0}^{j-1} Z_{t-k} .
$$

Our process of interest is the sum of a finite number of processes each with summable MA coefficients and thus its MA coefficients are absolutely summable. Lemma A-1 implies that uniformly over $\mathfrak{R}_{\delta}$

$$
B_{j, F Q D} \rightarrow^{p} E\left[\left(X_{t+j}+X_{t-j}-2 X_{t}\right) \varepsilon_{t}\right]=a_{j}
$$

Turning to (c), the object of interest is the $p \times p$ matrix:

$$
C_{j, F Q D}=\frac{1}{2 T} \sum_{t=j+p+1}^{T}\left(X_{t} X_{t-j}^{\prime}+X_{t-j} X_{t}^{\prime}-2 X_{t} X_{t}^{\prime}\right)
$$

where all elements except possibly the top-left element satisfy the uniform Law of Large Numbers, and thus are of order $O_{p}(1)$. Now the last $p-1$ elements of $X_{t}$ are $\Delta y_{t-1}, \ldots, \Delta y_{t-p+1}$. From Lemma S8 in Mikusheva (2007b), they have absolutely summable MA coefficients uniformly over $\mathfrak{R}_{\delta}$. Thus, the elements of the right-bottom $(p-1) \times(p-1)$ sub-matrix satisfy conditions of Lemma A-1. The elements in the first row and the first column (except the top-left element) are of the form

$$
\frac{1}{2 T} \sum_{t=j+p+1}^{T}\left(y_{t-1} z_{t-j}+y_{t-1-j} z_{t}-2 y_{t-1} z_{t}\right)=\frac{1}{2 T} \sum_{t=j+1}^{T}\left(y_{t-1+j}+y_{t-1-j}-2 y_{t-1}\right) z_{t}+O_{p}(1 / \sqrt{T}),
$$


where $z_{t}$ is one of $\Delta y_{t-1}, \ldots, \Delta y_{t-p+1}$. From the proof of (b), the series $y_{t-1+j}+y_{t-1-j}-2 y_{t-1}$ also has absolutely summable MA coefficients. Thus, the conditions of Lemma A-1 are satisfied.

It remains to consider the top-left element of the matrix $C_{j, F Q D}$ which is given by

$$
\left(C_{j, F Q D}\right)_{11}=\frac{1}{T} \sum_{t=j+p+1}^{T}\left[y_{t-1} y_{t-j-1}-y_{t-1}^{2}\right]
$$

If the largest (in absolute value) root $\lambda_{p}$ is not real, then by definition of $\mathfrak{R}_{\delta}$, it is less than $\delta<1$ in absolute value, and the process $y_{t}$ is uniformly stationary. Thus $\left(C_{j, F Q D}\right)_{11}$ satisfies the conditions of Lemma A-1. Assume now that the largest root $\lambda_{p}$ is a real number. We have $1-\alpha L-\sum_{j=1}^{p-1} b_{j} L^{j}(1-L)=\left(1-\lambda_{p}\right) B(L)$, where all inverse roots of $B(L)$ are strictly inside the circle of radius $\delta$.

Let $u_{t}=y_{t}-\lambda_{p} y_{t-1}$ and thus $B(L) u_{t}=\varepsilon_{t}$. Now $u_{t}$ has absolutely summable MA coefficients uniformly over $\mathfrak{R}_{\delta}$.

$$
\begin{aligned}
\frac{1}{T} \sum_{t=j+1}^{T} y_{t-1} y_{t-j-1} & =\frac{1}{T} \sum_{t=j+1}^{T} y_{t-j-1}\left(\lambda_{p}^{j} y_{t-j-1}+\sum_{k=0}^{j-1} \lambda_{p}^{k} u_{t-k-1}\right)= \\
& =\lambda_{p}^{j} \frac{1}{T} \sum_{t=1}^{T-j} y_{t-1}^{2}+\sum_{k=0}^{j-1} \lambda_{p}^{k} \frac{1}{T} \sum_{t=j+1}^{T} y_{t-j-1} u_{t-k-1} .
\end{aligned}
$$

As a result,

$$
\left(C_{j, F Q D}\right)_{11}=-\left(1-\lambda_{p}^{j}\right) \frac{1}{T} \sum_{t=1}^{T} y_{t-1}^{2}+\sum_{k=1}^{j-1} \lambda_{p}^{k-1} \frac{1}{T} \sum_{t=j+1}^{T} y_{t-j-1} u_{t-k-1}+O_{p}\left(T^{-1 / 2}\right),
$$

again the $O_{p}$ term appears due to change of summation bounds. First, observe that

$$
\begin{gathered}
\operatorname{Var}\left(\frac{1}{T} \sum_{t=1}^{T-j} y_{t} u_{t+k}\right)=\operatorname{Var}\left(\frac{1}{T} \sum_{t=1}^{T-j} \sum_{s=0}^{t} \lambda_{p}^{s} u_{t-s} u_{t+k}\right)= \\
=\frac{1}{T^{2}} \sum_{t=1}^{T-j} \sum_{s=0}^{t} \lambda_{p}^{s} \operatorname{cov}\left(u_{t+k}, u_{t-s}\right)<\operatorname{Var}\left(u_{t}\right)<\operatorname{const}(\delta) .
\end{gathered}
$$

The variance of $u_{t}$ is uniformly bounded because all roots of this process are uniformly separated from the unit circle. That is, $\frac{1}{T} \sum_{t=1}^{T-j} y_{t} u_{t+k}=O_{p}(1)$ uniformly over $\beta^{0} \in \mathfrak{R}_{\delta}$ and for all $1 \leq k \leq$ $j \leq K$.

Next, consider the term $\left(1-\lambda_{p}^{j}\right) \frac{1}{T} \sum_{t=1}^{T} y_{t-1}^{2}$. From Theorem 1 in Mikusheva (2011), $\left(1-\lambda_{p}\right) \frac{1}{T} \sum_{t=1}^{T} y_{t-1}^{2}$ is uniformly approximated by $\frac{\sigma^{2}}{g(c)} \int_{0}^{1} J_{c}^{2}(t) d t$, where $g(c)=E \int_{0}^{1} J_{c}^{2}(t) d t$, where $J_{c}(t)$ is the OrnsteinUhlenbeck process, and $c=T \log \left(\left|\lambda_{p}\right|\right)$. It follows from Lemma 4(h) and Lemma 10 in Mikusheva (2007a) that $\frac{1}{g(c)} \int_{0}^{1} J_{c}^{2}(t) d t$ is uniformly bounded in probability over $c$. Summing up, $C_{j, F Q D}$ is asymptotically uniformly $O_{p}(1)$ over $\mathfrak{R}_{\delta}$ and the proof of Lemma A-2 is complete. 
Lemma A-3 Under assumptions of Proposition 3 the estimator $\widehat{\beta}_{K, F Q D}$ is consistent for any $K>p$.

Proof of Lemma A-3 Let $f(x)=\left(f_{1}(x), \ldots, f_{p+1}(x)\right)$, where $f_{j}(x)=x^{\prime} B_{j, F Q D}+x^{\prime} C_{j, F Q D} x$ and $Q(x)=\sum_{j=1}^{K} f_{j}^{2}(x)$. Any $K \geq p+1$ suffices for consistency of $\widehat{\beta}_{K, F Q D}$, though additional moments may improve efficiency. For any bounded set $C$ in the parameter space, and by Lemma A-2, it holds that:

$$
\sup _{\beta \in C}\left|Q\left(\beta^{0}-\beta\right)-\sum_{j=1}^{K}\left(\bar{g}_{j, F Q D}(\beta)\right)^{2}\right|=o_{p}(1) .
$$

Since $Q(0)=0$, for consistency of $\widehat{\beta}_{K, F Q D}$, it is enough to show that for any $\varsigma>0$, there is $\varepsilon>0$ such that

$$
\lim _{T \rightarrow \infty} P\left(\inf _{|x|>\varsigma} Q(x)>\varepsilon\right)=1
$$

where $x=\beta^{0}-\beta$. Since $\beta^{0} \in \mathfrak{R}_{\delta}$ and $\beta$ belongs to bounded neighborhood of $\mathfrak{R}_{\delta}, x$ is bounded. There are two cases to consider: $\left|\lambda_{p}\right|<\delta_{1}<1$, and $\lambda_{p} \geq \delta_{1}$.

Case (i) $\left|\lambda_{p}\right|<\delta_{1}$ : We will show that for any fixed $0<\delta_{1}<1$ statement (A.8) holds uniformly over $\beta^{0} \in \mathfrak{R}_{\delta} \cap\left\{\left|\lambda_{p}\right|<\delta_{1}\right\}$.

Since $K>p, Q(x) \geq f(x)^{\prime} f(x)$. For any orthonormal transformation $A, Q(x) \geq(A f(x))^{\prime}(A f(x)) \geq$ $(A f(x))_{1}^{2}$, where $(A f)_{1}$ is the first component of vector $A f(x)$. Consider a linear transformation, the first component of which is

$$
(A f(x))_{1}=\left(f_{p+1}-\alpha^{0} f_{p}-\sum_{j=1}^{p-1} b_{j}^{0}\left(f_{p+1-j}-f_{p-j}\right)\right) \frac{1}{a}
$$

where $a=\sqrt{1+\left(\alpha^{0}+b_{1}^{0}\right)^{2}+\left(b_{2}^{0}-b_{1}^{0}\right)^{2}+\ldots}$ is a (non-zero) multiplier that normalizes the linear transformation. Let $\mathcal{A}(L)=1-\alpha^{0} L-\sum_{j=1}^{p-1} b_{j}^{0} L^{j}(1-L)$ be a lag operator. Given the definition of $f_{j}$ and linearity of the transformation,

$$
(A f(x))_{1}=\frac{1}{a} \mathcal{A}(L) f_{p+1}=\frac{1}{a}\left(x^{\prime}\left(\mathcal{A}(L) B_{p+1, F Q D}\right)+x^{\prime}\left(\mathcal{A}(L) C_{p+1, F Q D}\right) x\right) .
$$

From (b) of Lemma A-2, $B_{j, F Q D} \rightarrow^{p} a_{j}=E\left[X_{t+j} \varepsilon_{t}\right]$. From (10) and the definition of $X_{t}$,

$$
\mathcal{A}(L) X_{t+p+1}=\left[\varepsilon_{t+p+1}, \Delta \varepsilon_{t+p+1}, \ldots, \Delta \varepsilon_{t+2}\right]^{\prime}=\widetilde{e}_{t+p+1} .
$$

Since $E \widetilde{e}_{t+p+1} \varepsilon_{s}=0$ for any $s \leq t$,

$$
\mathcal{A}(L) B_{p+1, F Q D} \rightarrow^{p} \mathcal{A}(L) E\left[X_{t+j} \varepsilon_{t}\right]=E\left[\mathcal{A}(L) X_{t+j} \varepsilon_{t}\right]=E\left[\widetilde{e}_{t+p+1} \varepsilon_{t}\right]=0 .
$$

Thus, uniformly over all $x$ in a bounded set,

$$
(A f(x))_{1}=\frac{1}{a}\left(x^{\prime}\left(\mathcal{A}(L) C_{p+1, F Q D}\right) x\right)+o_{p}(1) .
$$


It follows from (13) and (A.10) that

$$
\mathcal{A}(L) C_{p+1, F Q D}=\frac{1}{2 T} \sum_{t=p+1}^{T}\left(\widetilde{e}_{t+p+1} X_{t}^{\prime}+X_{t} \widetilde{e}_{t+p+1}\right)-\mathcal{A}(1) \frac{1}{T} \sum_{t=p+1}^{T} X_{t} X_{t}^{\prime}+o_{p}(1),
$$

where $o_{p}(1)$ appears from change in the bounds of summation. Since $\left|\lambda_{p}\right|<\delta_{1}$ by assumption, the process is stationary. Thus $\frac{1}{2 T} \sum_{t=p+1}^{T} \widetilde{e}_{t+p+1} X_{t}^{\prime} \rightarrow^{p} 0$ uniformly over $\left|\lambda_{p}\right|<\delta_{1}$ and $\mathcal{A}(1) \frac{1}{T} \sum_{t=p+1}^{T} X_{t} X_{t}^{\prime}$ is uniformly positive definite. This gives us the needed bound in (A.8) for processes with $\left|\lambda_{p}\right|<\delta_{1}$.

Case (ii) $\left|\lambda_{p}\right| \geq \delta_{1}$ : To show that for $\delta_{1}<1$ close enough to the unity, (A.8) holds uniformly over $\beta^{0} \in \mathfrak{R}_{\delta} \cap\left\{\left|\lambda_{p}\right| \geq \delta_{1}\right\}$, we divide the area $|x|>\varsigma$ from (A.8) into two regions: $I_{1}=\{x:|x|>$ $\left.\varsigma,\left|x_{1}\right|>\varsigma_{1}\right\}$ and $I_{2}=\left\{x:|x|>\varsigma,\left|x_{1}\right| \leq \varsigma_{1}\right\}$, where $0<\varsigma_{1}<\varsigma$.

Consider $x \in I_{1}$. We will prove that for any fixed $\varsigma_{1}>0$, one can choose $\delta_{1}$ close enough to the unity such that uniformly over $\beta^{0} \in \mathfrak{R}_{\delta} \cap\left\{\left|\lambda_{p}\right|>\delta_{1}\right\}$, an analog of (A.8) holds where the infinimum is taken over $x \in I_{1}$.

Applying the arguments and transformation as in (A.9), it can be shown that equations (A.11) and (A.12) hold. Since $\mathcal{A}(1)$ converges to zero as $\delta_{1}$ converges to 1 , one can choose $\delta_{1}$ close enough to 1 to make all terms except the $(1,1)$-th element of $\mathcal{A}(1) \frac{1}{T} \sum_{t=p+1}^{T} X_{t} X_{t}^{\prime}$ sufficiently small, and all but the $(1,1)$-th element of $\frac{1}{T} \sum_{t=p+1}^{T} \widetilde{e}_{t+p+1} X_{t}^{\prime}$ converge in probability to its expected value of zero. In consequence, the following holds uniformly over $\beta^{0} \in \mathfrak{R}_{\delta} \cap\left\{\left|\lambda_{p}\right|>\delta_{1}\right\}$ and $x \in I_{1}$ :

$$
x^{\prime} \mathcal{A}(L) C_{p+1, F Q D} x=x_{1}^{2}\left(\frac{1}{T} \sum_{t=p+1}^{T} \varepsilon_{t+p+1} y_{t-1}-\mathcal{A}(1) \frac{1}{T} \sum_{t=p+1}^{T} y_{t-1}^{2}\right)+o_{p}(1)+o_{p}\left(1-\delta_{1}\right) .
$$

It remains to show that $\frac{1}{T} \sum_{t=p+1}^{T}\left(\varepsilon_{t+p+1}-\mathcal{A}(1) y_{t-1}\right) y_{t-1}$ satisfies the uniform Law of Large Numbers, and thus converges uniformly to a non-zero constant. To do so, we use the decomposition as in Phillips and Solo (1992)) that $\varepsilon_{t+p+1}-\mathcal{A}(1) y_{t-1}=u_{t}-u_{t-1}$, where $u_{t}$ is a series with absolutely summable MA coefficients. Since $\frac{1}{T}\left(u_{t}-u_{t-1}\right) y_{t-1}=-\frac{1}{T}\left(y_{t}-y_{t-1}\right) u_{t}+O_{p}(1 / \sqrt{T})$, Lemma A-1 applies, and $\frac{1}{T} \sum_{t=p+1}^{T}\left(\varepsilon_{t+p+1}-\mathcal{A}(1) y_{t-1}\right) y_{t-1}$ converges in probability to its expectation. Since $\frac{\mathcal{A}(1)}{T} E \sum_{t=p+1}^{T} y_{t-1}^{2}$ is uniformly different from zero, this implies that for any fixed $\varsigma_{1}>0$ there exists $\delta_{1}<1$ such that uniformly over $\left|\lambda_{p}\right|>\delta_{1}$ an analog of (A.8) holds where the infinimum is taken over $x \in I_{1}$.

Consider now $x \in I_{2}$. One can choose $\varsigma_{1}$ small enough and $\delta_{1}$ close enough to the unity such that uniformly over $\beta^{0} \in \mathfrak{R}_{\delta} \cap\left\{\left|\lambda_{p}\right|>\delta_{1}\right\}$, an analog of (A.8) holds, where the infinimum is taken over $x \in I_{2}$. Given that $B_{j, F Q D}$ and $C_{j, F Q D}$ are uniformly bounded,

$$
f_{j}(x)=x_{-1}^{\prime} B_{j,-1}+x_{-1}^{\prime} C_{j,-1} x_{-1}+o_{p}\left(\varsigma_{1}\right) .
$$

where $x_{-1}=\left(x_{2}, \ldots, x_{p}\right)$ is $(p-1) \times 1$ sub-vector of $x$, and $B_{j,-1}$ and $C_{j,-1}$ are the $(p-1) \times 1$ and $(p-1) \times(p-1)$ sub-matrixes of $B_{j, F Q D}$ and $C_{j, F Q D}$ corresponding to the last $p-1$ components of $\beta$. 
Let $Z_{t}=\left(\Delta y_{t-1}, \ldots, \Delta y_{t-p+1}\right)$ and $\widetilde{Z}_{t}=\left(y_{t-1}-\lambda_{p} y_{t-2}, \ldots, y_{t-p+1}-\lambda_{p} y_{t-p}\right)^{\prime}$ be two $(p-1) \times 1$ uniformly stationary vector-processes. Note that the matrices $B_{j,-1}$ and $C_{j,-1}$ satisfy equations analogous to (12) and (13) with $Z_{t}$ in place of $X_{t}$. Similarly, $\widetilde{B}_{j}$ and $\widetilde{C}_{j}$ are defined as in (12) and (13) with $\widetilde{Z}_{t}$ in place of $X_{t}$. Observe that $Z_{t}^{\prime}=\widetilde{Z}_{t}^{\prime}-\left(1-\lambda_{p}\right)\left(y_{t-2}, \ldots, y_{t-p}\right)$. It is easy to see that

$$
f_{j}(x)=x_{-1}^{\prime} \widetilde{B}_{j}+x_{-1}^{\prime} \widetilde{C}_{j} x_{-1}+o_{p}\left(1-\delta_{1}\right)+o_{p}\left(\varsigma_{1}\right) .
$$

The function $\widetilde{f}_{j}=x_{-1}^{\prime} \widetilde{B}_{j}+x_{-1}^{\prime} \widetilde{C}_{j} x_{-1}$ corresponds to that of the uniformly stationary process $y_{t}-\lambda_{p} y_{t-1}$ with all roots smaller than $\delta$ in absolute value. The rest of the proof follows arguments as in Case i.

Proof of Proposition 3 (i): $\quad$ To establish the asymptotic distribution of $\widehat{\beta}_{K, F Q D}$, consider first order condition:

$$
\sum_{j=1}^{K} \bar{g}_{j, F Q D}\left(\widehat{\beta}_{K, F Q D}\right) \frac{\partial \bar{g}_{j, F Q D}}{\partial \beta}\left(\widehat{\beta}_{K, F Q D}\right)=0 .
$$

From Lemma A-2 and consistency of $\widehat{\beta}_{K, F Q D}$,

$$
\frac{\partial \bar{g}_{j, F Q D}}{\partial \beta}\left(\widehat{\beta}_{K, F Q D}\right)=-B_{j, F Q D}+o_{p}(1) \rightarrow^{p} a_{j},
$$

and uniformly over $\mathfrak{R}_{\delta}$ :

$$
\sqrt{T} \bar{g}_{j, F Q D}\left(\widehat{\beta}_{K, F Q D}\right)=\sqrt{T} A_{j, F Q D}+a_{j}^{\prime} \sqrt{T}\left(\widehat{\beta}_{K, F Q D}-\beta^{0}\right)+o_{p}(1) .
$$

As a result, the following holds uniformly:

$$
\sqrt{T}\left(\widehat{\beta}_{K, F Q D}-\beta^{0}\right) \Rightarrow\left(\sum_{j=1}^{K} a_{j} a_{j}^{\prime}\right)^{-1}\left(\sum_{j=1}^{K} a_{j}\left(\xi_{j}-\xi_{0}\right)\right)=N\left(0, \Sigma_{K, F Q D}\right),
$$

where $G=\left(\sum_{j=1}^{K} a_{j} a_{j}^{\prime}\right)^{-1}$, and $\Sigma_{K, F Q D}=\sigma^{4} G+\mu_{4} G\left(\sum_{j=1}^{K} a_{j}\right)\left(\sum_{j=1}^{K} a_{j}\right)^{\prime} G$.

Proof of Proposition 3 (ii): The proof proceeds by treating QD as a two-step estimator. First, note that

$$
s^{2}-\frac{1}{T} \sum_{t=p+1}^{T} \varepsilon_{t}^{2}=-\frac{1}{T}\left(\sum_{t} X_{t} \varepsilon_{t}\right)^{\prime}\left(\sum_{t} X_{t} X_{t}^{\prime}\right)^{-1}\left(\sum_{t} X_{t} \varepsilon_{t}\right)
$$

Theorem 1 in Mikusheva (2011) shows that the statistic $\left(\sum_{t} X_{t} \varepsilon_{t}\right)^{\prime}\left(\sum_{t} X_{t} X_{t}^{\prime}\right)^{-1}\left(\sum_{t} X_{t} \varepsilon_{t}\right)$ is uniformly approximated by the distribution $\left(t^{c}+N(0, p-1)\right)^{2}$, where $t^{c}=\frac{\int J_{c}(t) d w(t)}{\sqrt{\int J_{c}^{2}(t) d t}}$ is a local-to-unity limit of a t-statistic, and $c=T \log \left(\left|\lambda_{p}\right|\right)$. Given that $t^{c}$ is uniformly bounded in probability over all possible values of $c \leq 0$, the following holds uniformly over $\mathfrak{R}_{\delta}$ :

$$
s^{2}=\frac{1}{T} \sum_{t} \varepsilon_{t}^{2}+O_{p}(1 / T)
$$


Since $\bar{g}_{j, Q D}(\beta)=\bar{g}_{j, F Q D}(\beta)-\gamma_{0}+s^{2}$,

$$
\bar{g}_{j, Q D}(\beta)=A_{j, Q D}+\left(\beta^{0}-\beta\right)^{\prime} B_{j, Q D}+\left(\beta^{0}-\beta\right)^{\prime} C_{j, Q D}\left(\beta^{0}-\beta\right),
$$

where $A_{j, Q D}=A_{j, F Q D}+s^{2}-\sigma^{2}, B_{j, Q D}=B_{j, F Q D}$ and $C_{j, Q D}=C_{j, F Q D}$. A result analogous of Lemma A-2 holds for $A_{j, Q D}, B_{j, Q D}$ and $C_{j, Q D}$ with one correction: $\sqrt{T}\left(A_{1, Q D}, \ldots, A_{K, Q D}\right) \Rightarrow$ $\left(\xi_{1}, \ldots, \xi_{K}\right)$. This gives us consistency and asymptotic normality of $\widehat{\beta}_{K, Q D}$ with asymptotic covariance matrix $\Sigma_{K, Q D}=\sigma^{4} G$.

The following lemma will be used to prove Proposition 4.

Lemma A-4 Uniformly over all possible values of $\theta$,

(a) $\sqrt{T}\left(A_{j}+\Omega^{0}\right) \Rightarrow \xi_{j}-\xi_{0}$;

(b) $\sqrt{T}\left(A_{j}+S\right) \Rightarrow \xi_{j}$;

(c) $B_{j, 1} \rightarrow^{p} E\left[\varepsilon_{t}\left(x_{t-j-1}-x_{t-1}\right)\right]=0$;

(d) $B_{j, 2} \rightarrow^{p} E\left[x_{t-1}\left(\varepsilon_{t-j}-\varepsilon_{t}\right)^{\prime}\right]=a_{j}$;

(e) $C_{j}=O_{p}(1)$.

where $\frac{1}{\sqrt{T}} \sum_{t=j+1}^{T} \varepsilon_{t} \varepsilon_{t-j}^{\prime} \Rightarrow \xi_{j}$ and $\xi_{j}$ is a $2 \times 2$ matrix with normally distributed components such that for any non-random vector a the vector $\xi_{j} \mathbf{a}$ is normally distributed with variance-covariance matrix $\Omega^{0} \mathbf{a}^{\prime} \Omega^{0} \mathbf{a}$. We also have $\frac{1}{\sqrt{T}} \sum_{t=j+1}^{T} \varepsilon_{t} \varepsilon_{t}^{\prime} \Rightarrow \xi_{0}$ where $\xi_{0}$ is a $2 \times 2$ matrix with normally distributed components such that for any non-random vector $\mathbf{a}$, the vector $\xi_{0} \mathbf{a}$ is normally distributed with variance-covariance matrix $E\left[\left(\varepsilon_{t}^{\prime} \mathbf{a}\right)^{2} \varepsilon_{t} \varepsilon_{t}^{\prime}\right]$. The variables $\xi_{j}$ are independent for any $j \geq 0$.

Proof of Lemma A-4 Result (a) follows from Central Limit Theorem. To prove (b), note that

$$
S=\frac{1}{T} \sum_{t=1}^{T} \varepsilon_{t} \varepsilon_{t}^{\prime}-\frac{1}{T} \frac{1}{\sum_{s} x_{s-1}^{2}}\left(\begin{array}{cc}
\left(\sum_{s} \varepsilon_{x s} x_{s-1}\right)^{2} & \left(\sum_{s} \varepsilon_{x s} x_{s-1}\right)\left(\sum_{s} \varepsilon_{y s} x_{s-1}\right) \\
\left(\sum_{s} \varepsilon_{y s} x_{s-1}\right)\left(\sum_{s} \varepsilon_{x s} x_{s-1}\right) & \left(\sum_{s} \varepsilon_{y s} x_{s-1}\right)^{2}
\end{array}\right)
$$

Now $\frac{\sum_{s=1}^{T} \varepsilon_{x s} x_{s-1}}{\sqrt{\sum_{s=1}^{T} x_{s-1}^{2}}} \Rightarrow t^{c}$ uniformly over $\alpha^{0} \in(-1+\delta, 1]$, and the family $t^{c}$ is uniformly bounded. The $\operatorname{sum} \frac{\sum_{s=1}^{T} \varepsilon_{y s} x_{s-1}}{\sqrt{\sum_{s=1}^{T} E x_{s-1}^{2}}}$ has a bounded second moment since $\varepsilon_{y s} x_{s-1}$ is martingale-difference sequence, and thus it is uniformly bounded by Chebyshev's inequality. Lastly, $\frac{\sum_{s=1}^{T} x_{s-1}^{2}}{\sum_{s=1}^{T} E x_{s-1}^{2}}$ is uniformly separated from zero, a result that follows from Lemma 4(h) and Lemma 10 in Mikusheva (2007a). Summing up, we have $S=\frac{1}{T} \sum_{t=1}^{T} \varepsilon_{t} \varepsilon_{t}^{\prime}+O_{p}\left(\frac{1}{T}\right)$. As a result, $\sqrt{T}\left(A_{j}+S\right)=\frac{1}{\sqrt{T}} \sum_{t=j+1}^{T} \varepsilon_{t} \varepsilon_{t-j}^{\prime} \Rightarrow \xi_{j}$.

The proof of part (c) follows from Lemma A-1, since we show in the proof of Proposition 3 that $x_{t-j-1}-x_{t-1}$ has absolutely summable MA coefficients uniformly over $\alpha$. 
To prove (d), re-write

$$
B_{j, 2}=\frac{1}{T} \sum_{t=j+1}^{T} x_{t-1}\left(\varepsilon_{t-j}-\varepsilon_{t}\right)^{\prime}=\frac{1}{T} \sum_{t=j+1}^{T}\left(x_{t+j-1}-x_{t-1}\right) \varepsilon_{t}^{\prime}+\frac{1}{T} \sum_{t=j+1}^{2 j} x_{t-1} \varepsilon_{t-j}-\frac{1}{T} \sum_{t=T+1}^{T+j} x_{t-1} \varepsilon_{t-j} .
$$

The terms $\frac{1}{T} \sum_{t=j+1}^{2 j} x_{t-1} \varepsilon_{t-j}$ and $\frac{1}{T} \sum_{t=T+1}^{T+j} x_{t-1} \varepsilon_{t-j}$ both have $j$ summands each of which are of order $O_{p}(\sqrt{T})$. This means that for any $j \leq K$ where $K$ is fixed, the following holds uniformly:

$$
B_{j, 2}=\frac{1}{T} \sum_{t=j+1}^{T}\left(x_{t+j-1}-x_{t-1}\right) \varepsilon_{t}^{\prime}+O_{p}\left(\frac{1}{\sqrt{T}}\right) .
$$

The rest of the proof is the same as for part (c). Part (e) follows from Proposition 3.(c).

Proof of Proposition 4: Note first that

$$
\begin{aligned}
\widehat{\Gamma}_{j}(\theta)-\widehat{\Gamma}_{0}(\theta) & =\frac{1}{T} \sum_{t=j+1}^{T} e_{t}(\theta)\left(e_{t-j}(\theta)-e_{t}(\theta)\right)^{\prime} \\
& =\frac{1}{T} \sum_{t=j+1}^{T}\left(\left(\theta^{0}-\theta\right) x_{t-1}+\varepsilon_{t}\right)\left(\left(\theta^{0}-\theta\right)\left(x_{t-j-1}-x_{t-1}\right)+\varepsilon_{t-j}-\varepsilon_{t}\right)^{\prime} \\
& =A_{j}+B_{j, 1}\left(\theta^{0}-\theta\right)^{\prime}+\left(\theta^{0}-\theta\right) B_{j, 2}+\left(\theta^{0}-\theta\right) C_{j}\left(\theta^{0}-\theta\right)^{\prime}
\end{aligned}
$$

where

$$
\begin{array}{r}
A_{j}=\frac{1}{T} \sum_{t=j+1}^{T} \varepsilon_{t}\left(\varepsilon_{t-j}-\varepsilon_{t}\right)^{\prime} ; B_{j, 1}=\frac{1}{T} \sum_{t=j+1}^{T} \varepsilon_{t}\left(x_{t-j-1}-x_{t-1}\right) ; \\
B_{j, 2}=\frac{1}{T} \sum_{t=j+1}^{T} x_{t-1}\left(\varepsilon_{t-j}-\varepsilon_{t}\right)^{\prime} ; C_{j}=\frac{1}{T} \sum_{t=j+1}^{T} x_{t-1}\left(x_{t-j-1}-x_{t-1}\right) .
\end{array}
$$

Lemma A-4 showed that uniformly over $\alpha$ :

$$
\left\|\widehat{\Gamma}_{j}(\theta)-\widehat{\Gamma}_{0}(\theta)+\Omega^{0}\right\|_{2}^{2}=\left\|\left(\theta^{0}-\theta\right) B_{j, 2}+C_{j}\left(\theta^{0}-\theta\right)\left(\theta^{0}-\theta\right)^{\prime}\right\|_{2}^{2}+o_{p}(1),
$$

and

$$
\left\|\widehat{\Gamma}_{j}(\theta)-\widehat{\Gamma}_{0}(\theta)+S\right\|_{2}^{2}=\left\|\left(\theta^{0}-\theta\right) B_{j, 2}+C_{j}\left(\theta^{0}-\theta\right)\left(\theta^{0}-\theta\right)^{\prime}\right\|_{2}^{2}+o_{p}(1)
$$

We minimize the sum of such functions for $j=1, \ldots, K$. Obviously, the minimized function is nonnegative and one of its minimal value of zero is achieved at $\theta=\theta^{0}$. The question is whether there are any other minima. For this, there should exist $\theta$ such that $\left\|\left(\theta^{0}-\theta\right) B_{j, 2}+C_{j}\left(\theta^{0}-\theta\right)\left(\theta^{0}-\theta\right)^{\prime}\right\|_{2}$ is zero for all $j$. For a given $j$, the only non-trivial null of function $\left\|\left(\theta^{0}-\theta\right) B_{j, 2}+C_{j}\left(\theta^{0}-\theta\right)\left(\theta^{0}-\theta\right)^{\prime}\right\|_{2}$ implies $\theta_{j}=\theta^{0}+\frac{1}{C_{j}} B_{j, 2}$ which is asymptotically different for different $j$. This implies that for $K \geq 2$ no other asymptotic null of the objective function other than $\theta=\theta^{0}$ exists, and thus $\widehat{\theta}_{K, F Q D}$ and $\widehat{\theta}_{K, Q D}$ are consistent. 
To derive the limit distribution of $\widehat{\theta}_{K, F Q D}$, we use the fact that the first order condition must be satisfied at $\theta=\widehat{\theta}_{K, F Q D}$. Now the first order condition is

$$
\begin{aligned}
& \left.\nabla_{\theta} \| \widehat{\Gamma}_{j}(\theta)\right)-\widehat{\Gamma}_{0}(\theta)+\Omega^{0} \|_{2}^{2} \\
= & -2\left(A_{j}+\Sigma^{0}+B_{j, 1}\left(\theta^{0}-\theta\right)^{\prime}+\left(\theta^{0}-\theta\right) B_{j, 2}+C_{j}\left(\theta^{0}-\theta\right)\left(\theta^{0}-\theta\right)^{\prime}\right)\left(B_{j, 1}^{\prime}+B_{j, 2}+2 C_{j}\left(\theta^{0}-\theta\right)^{\prime}\right)^{\prime} .
\end{aligned}
$$

Since we proved that $\widehat{\theta}_{K, F Q D}$ is uniformly consistent, and given statements (d) and (e),

$$
B_{j, 2}+2 C_{j}\left(\theta^{0}-\widehat{\theta}_{K, F Q D}\right)^{\prime} \rightarrow^{p} a_{j} .
$$

Furthermore,

$$
\begin{aligned}
& \sqrt{T}\left(A_{j}+\Omega^{0}+B_{j, 1}\left(\theta^{0}-\widehat{\theta}_{K, F Q D}\right)+\left(\theta^{0}-\widehat{\theta}_{K, F Q D}\right) B_{j, 2}+C_{j}\left(\theta^{0}-\widehat{\theta}_{K, F Q D}\right)\left(\theta^{0}-\widehat{\theta}_{K, F Q D}\right)^{\prime}\right) \\
& =\sqrt{T}\left(A_{j}+\Omega^{0}\right)+\sqrt{T}\left(\theta^{0}-\widehat{\theta}_{K, F Q D}\right) a_{j}+o_{p}(1) .
\end{aligned}
$$

As a result,

$$
\sqrt{T}\left(\widehat{\theta}_{K, F Q D}-\theta^{0}\right) \Rightarrow \frac{1}{\sum_{j=1}^{K} a_{j} a_{j}^{\prime}} \sum_{j=1}^{K}\left(\xi_{j}-\xi_{0}\right) a_{j}
$$

uniformly over $\alpha$. Similarly,

$$
\sqrt{T}\left(\widehat{\theta}_{K, Q D}-\theta^{0}\right) \Rightarrow \frac{1}{\sum_{j=1}^{K} a_{j} a_{j}^{\prime}} \sum_{j=1}^{K} \xi_{j} a_{j} .
$$

The last two formulas lead to the conclusion of Lemma 4.

Relation between PH and FD: Observe that

$$
\begin{aligned}
\sum_{t=2}^{T}\left(\Delta y_{t-1}\right)^{2} & =\sum_{t=2}^{T} \Delta y_{t-1} y_{t-1}-\sum_{t=2}^{T}\left(y_{t-1}-y_{t-2}\right) y_{t-2} \\
& =\sum_{t=2}^{T} \Delta y_{t-1} y_{t-1}+\sum_{t=2}^{T} y_{t-1}\left(y_{t-1}-y_{t-2}\right)-y_{T-1}^{2}+y_{0}^{2}=2 \sum_{t=2}^{T} \Delta y_{t-1} y_{t-1}-y_{T-1}^{2}+y_{0}^{2} .
\end{aligned}
$$

Thus if $\left|\alpha^{0}\right|<1$ is fixed and $T \rightarrow \infty$,

$$
\frac{1}{T} \sum_{t=2}^{T}\left(\Delta y_{t-1}\right)^{2}=2 \frac{1}{T} \sum_{t=2}^{T} \Delta y_{t-1} y_{t-1}+O_{p}(1 / T) .
$$

Similarly,

$$
\begin{aligned}
\sum_{t=2}^{T} \Delta y_{t-1}\left(2 \Delta y_{t}+\Delta y_{t-1}\right) & =2 \sum_{t=2}^{T} \Delta y_{t-1} y_{t}-2 \sum_{t=2}^{T} \Delta y_{t-1} y_{t-1}+\sum_{t=2}^{T}\left(\Delta y_{t-1}\right)^{2}= \\
& =2 \sum_{t=2}^{T} \Delta y_{t-1} y_{t}-y_{T-1}^{2}+y_{0}^{2}
\end{aligned}
$$


and

$$
\frac{1}{T} \sum_{t=2}^{T} \Delta y_{t-1}\left(2 \Delta y_{t}+\Delta y_{t-1}\right)=2 \frac{1}{T} \sum_{t=2}^{T} \Delta y_{t-1} y_{t}+O_{p}(1 / T) .
$$

This leads us to the result that $\widehat{\alpha}_{P H}=\widehat{\alpha}_{F D}+O_{p}\left(T^{-1}\right)$ under stationary asymptotics. 


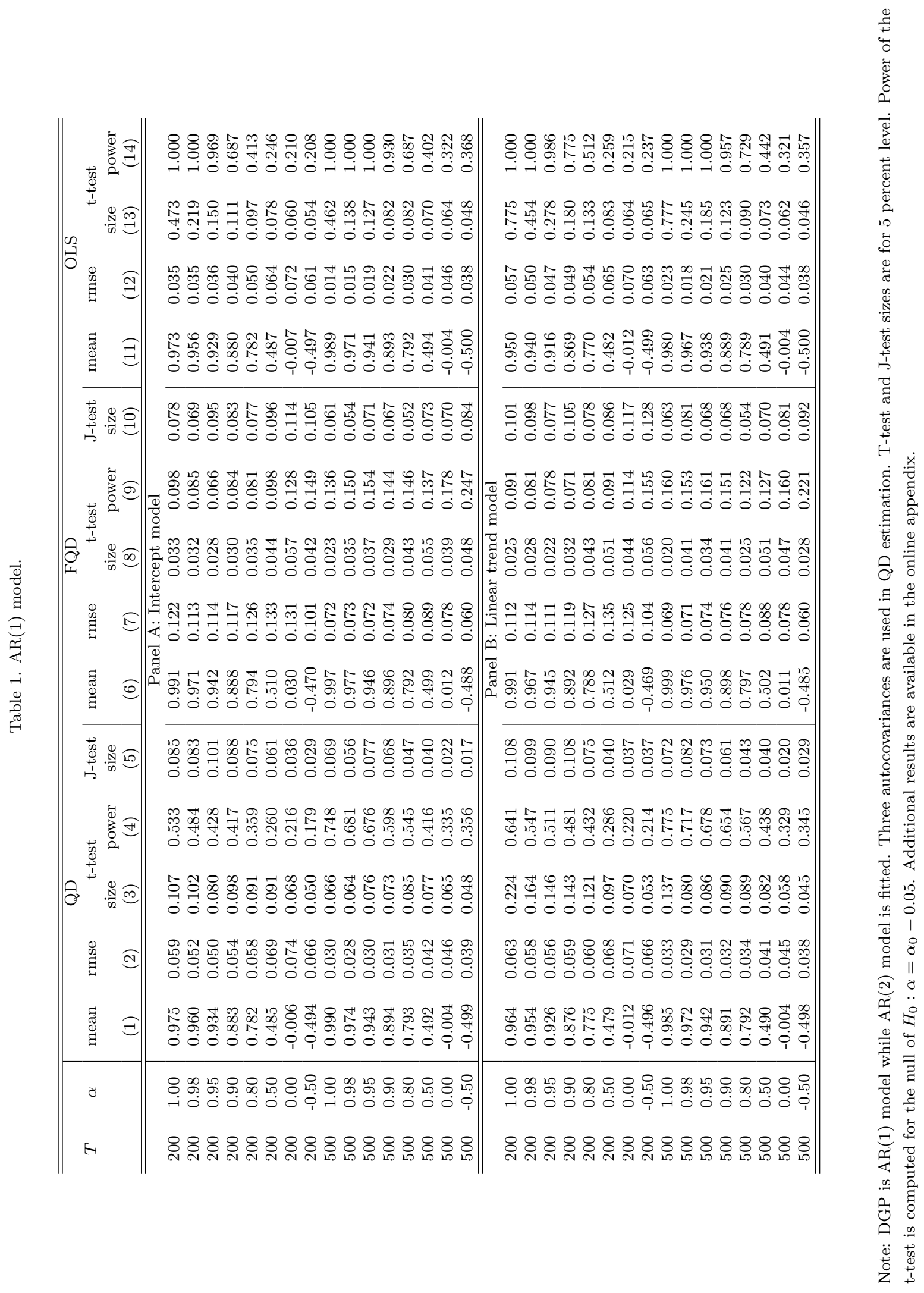




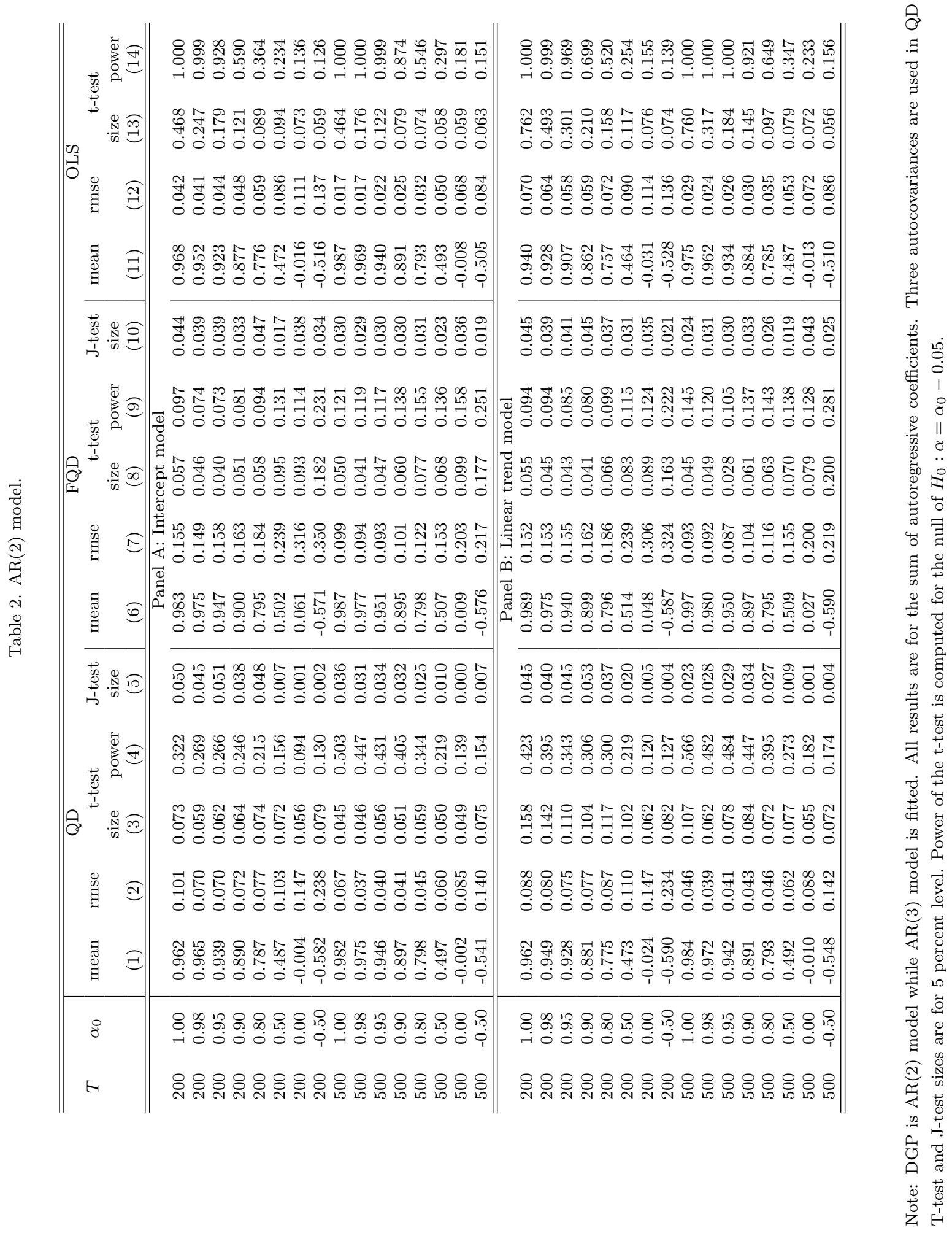




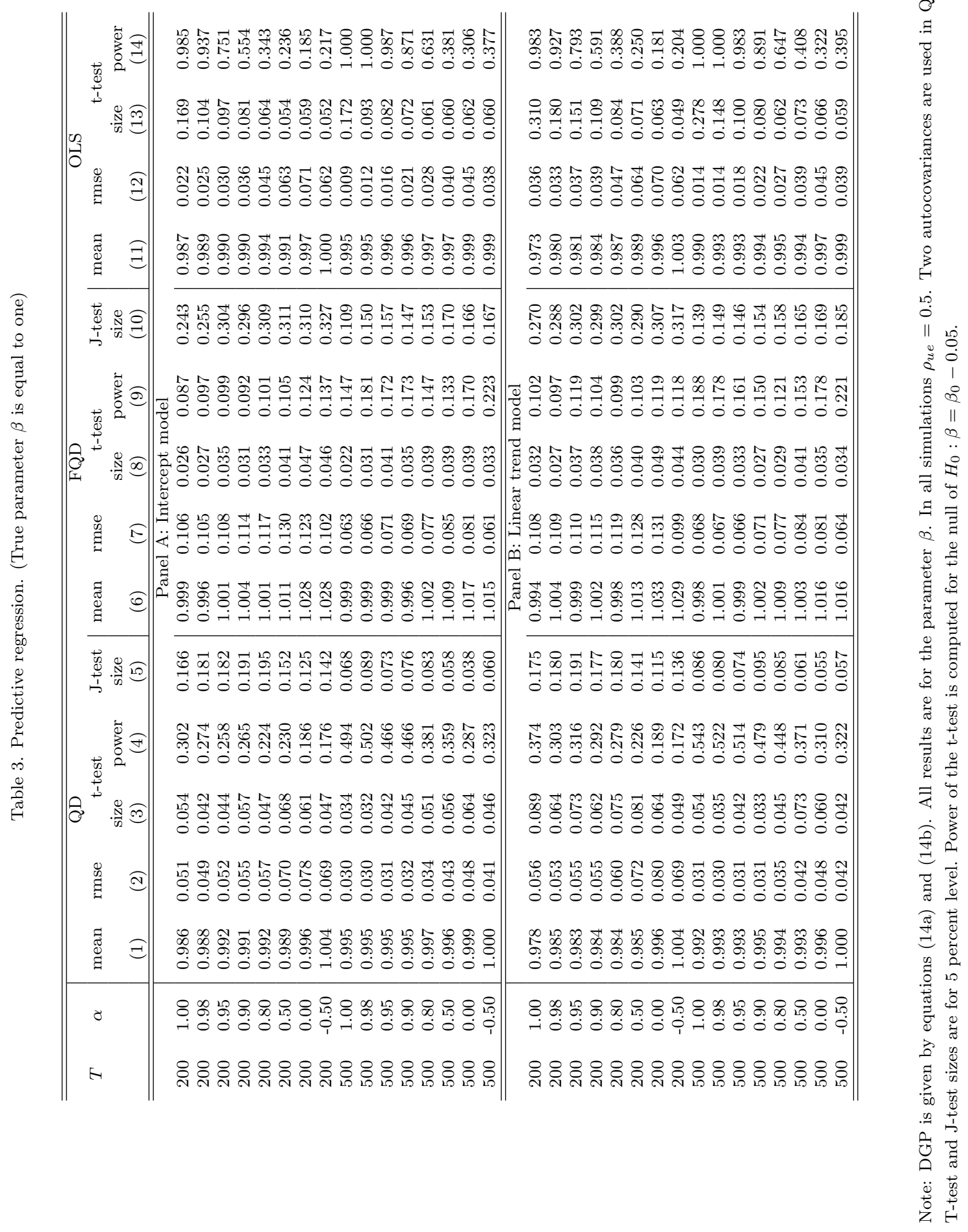


Table 4. DSGE model, capital intensity $\psi$.

\begin{tabular}{|c|c|c|c|c|c|c|c|c|c|c|}
\hline \multirow{3}{*}{$T$} & \multirow{3}{*}{$\rho$} & \multicolumn{5}{|c|}{ QD } & \multicolumn{4}{|c|}{ MLE } \\
\hline & & \multirow{2}{*}{$\begin{array}{c}\text { mean } \\
(1)\end{array}$} & \multirow{2}{*}{$\begin{array}{l}\text { rmse } \\
(2) \\
\end{array}$} & \multicolumn{2}{|c|}{ t-test } & \multirow{2}{*}{$\begin{array}{c}\text { J-test } \\
\text { size } \\
(5)\end{array}$} & \multirow{2}{*}{$\begin{array}{c}\text { mean } \\
(6)\end{array}$} & \multirow{2}{*}{$\begin{array}{l}\text { rmse } \\
(7) \\
\end{array}$} & \multicolumn{2}{|c|}{ t-test } \\
\hline & & & & $\begin{array}{c}\text { size } \\
(3) \\
\end{array}$ & $\begin{array}{c}\text { power } \\
(4)\end{array}$ & & & & $\begin{array}{c}\text { size } \\
(8) \\
\end{array}$ & $\begin{array}{c}\text { power } \\
(9) \\
\end{array}$ \\
\hline \multicolumn{11}{|c|}{ Panel A: Intercept model } \\
\hline 200 & 1.00 & 0.255 & 0.122 & 0.077 & 0.245 & 0.088 & 0.181 & 0.117 & 0.309 & 0.577 \\
\hline 200 & 0.98 & 0.255 & 0.120 & 0.098 & 0.259 & 0.085 & 0.220 & 0.094 & 0.142 & 0.409 \\
\hline 200 & 0.95 & 0.268 & 0.133 & 0.076 & 0.238 & 0.095 & 0.246 & 0.094 & 0.076 & 0.294 \\
\hline 200 & 0.90 & 0.284 & 0.147 & 0.066 & 0.201 & 0.094 & 0.266 & 0.114 & 0.065 & 0.235 \\
\hline 200 & 0.80 & 0.289 & 0.144 & 0.046 & 0.148 & 0.079 & 0.285 & 0.131 & 0.065 & 0.184 \\
\hline 500 & 1.00 & 0.260 & 0.072 & 0.050 & 0.304 & 0.049 & 0.173 & 0.110 & 0.517 & 0.830 \\
\hline 500 & 0.98 & 0.261 & 0.071 & 0.059 & 0.322 & 0.053 & 0.231 & 0.058 & 0.151 & 0.626 \\
\hline 500 & 0.95 & 0.262 & 0.073 & 0.066 & 0.331 & 0.049 & 0.245 & 0.054 & 0.076 & 0.522 \\
\hline 500 & 0.90 & 0.266 & 0.082 & 0.052 & 0.306 & 0.043 & 0.253 & 0.062 & 0.053 & 0.428 \\
\hline 500 & 0.80 & 0.281 & 0.110 & 0.035 & 0.197 & 0.035 & 0.270 & 0.096 & 0.062 & 0.315 \\
\hline \multicolumn{11}{|c|}{ Panel B: Linear trend model } \\
\hline 200 & 1.00 & 0.255 & 0.123 & 0.088 & 0.264 & 0.093 & 0.223 & 0.094 & 0.153 & 0.399 \\
\hline 200 & 0.98 & 0.267 & 0.128 & 0.075 & 0.222 & 0.090 & 0.243 & 0.091 & 0.102 & 0.315 \\
\hline 200 & 0.95 & 0.272 & 0.138 & 0.085 & 0.233 & 0.090 & 0.257 & 0.097 & 0.065 & 0.249 \\
\hline 200 & 0.90 & 0.282 & 0.145 & 0.066 & 0.204 & 0.083 & 0.273 & 0.113 & 0.057 & 0.205 \\
\hline 200 & 0.80 & 0.298 & 0.146 & 0.053 & 0.130 & 0.081 & 0.296 & 0.135 & 0.057 & 0.150 \\
\hline 500 & 1.00 & 0.259 & 0.071 & 0.050 & 0.308 & 0.041 & 0.209 & 0.079 & 0.324 & 0.730 \\
\hline 500 & 0.98 & 0.262 & 0.072 & 0.057 & 0.309 & 0.040 & 0.237 & 0.059 & 0.139 & 0.593 \\
\hline 500 & 0.95 & 0.263 & 0.073 & 0.058 & 0.315 & 0.039 & 0.250 & 0.056 & 0.069 & 0.477 \\
\hline 500 & 0.90 & 0.268 & 0.082 & 0.042 & 0.280 & 0.034 & 0.259 & 0.064 & 0.042 & 0.387 \\
\hline 500 & 0.80 & 0.284 & 0.108 & 0.024 & 0.162 & 0.028 & 0.277 & 0.097 & 0.053 & 0.295 \\
\hline
\end{tabular}

Note: The true value of capital intensity is $\psi=0.25$. The observed series is consumption. $Q D$ uses OLS estimate of the standard deviation of innovations consumption for QD estimation. $M L E$ corresponds to the maximum likelihood estimation (Kalman filter) of the structural parameters. Three autocorrelation coefficients (i.e, the fitted model is $\mathrm{AR}(3))$ and six autocovariances are used in QD estimation. T-test and J-test sizes are for 5 percent level. Power of the t-test is computed for the null of $H_{0}: \psi=\psi_{0}-0.1$. 


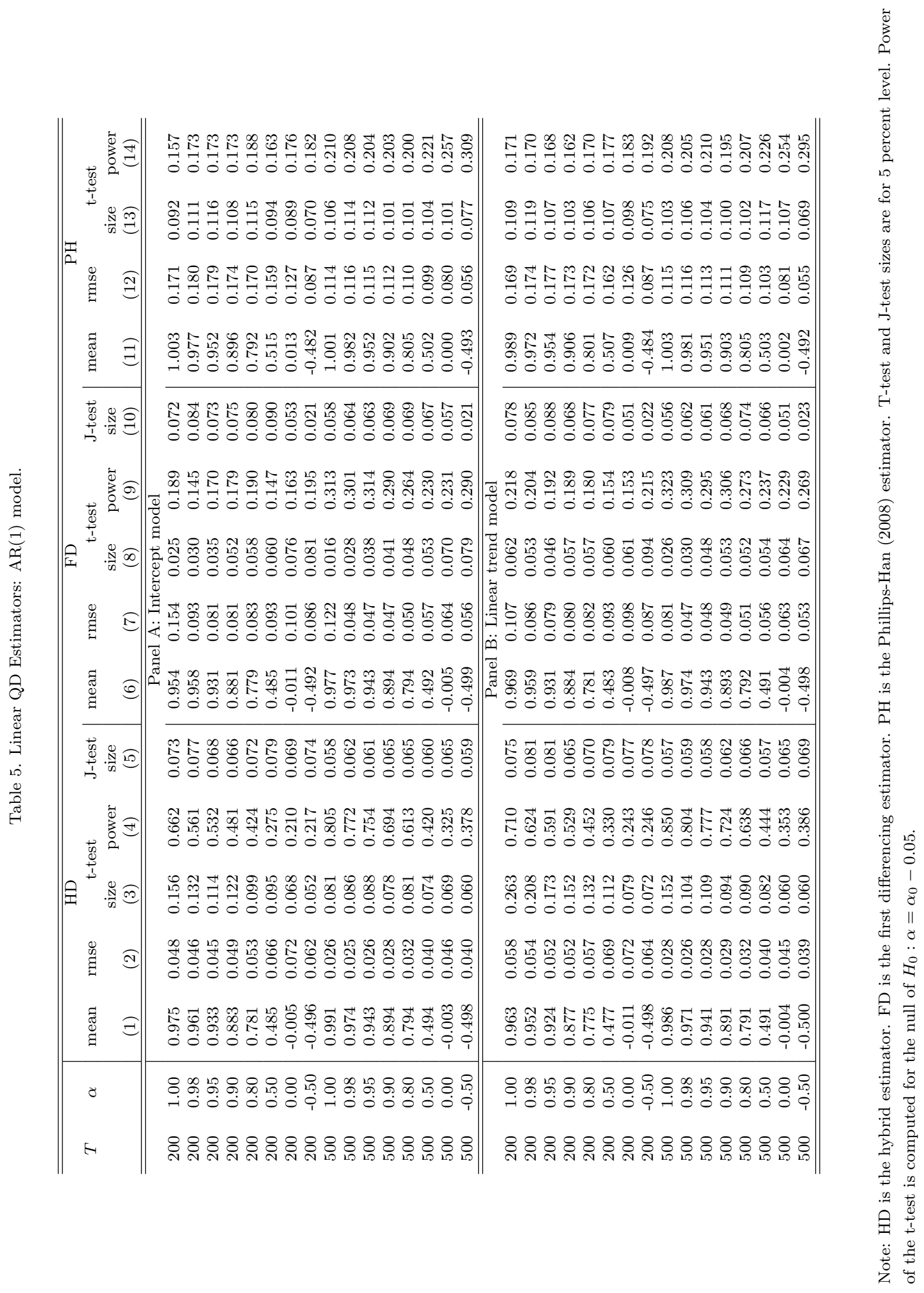



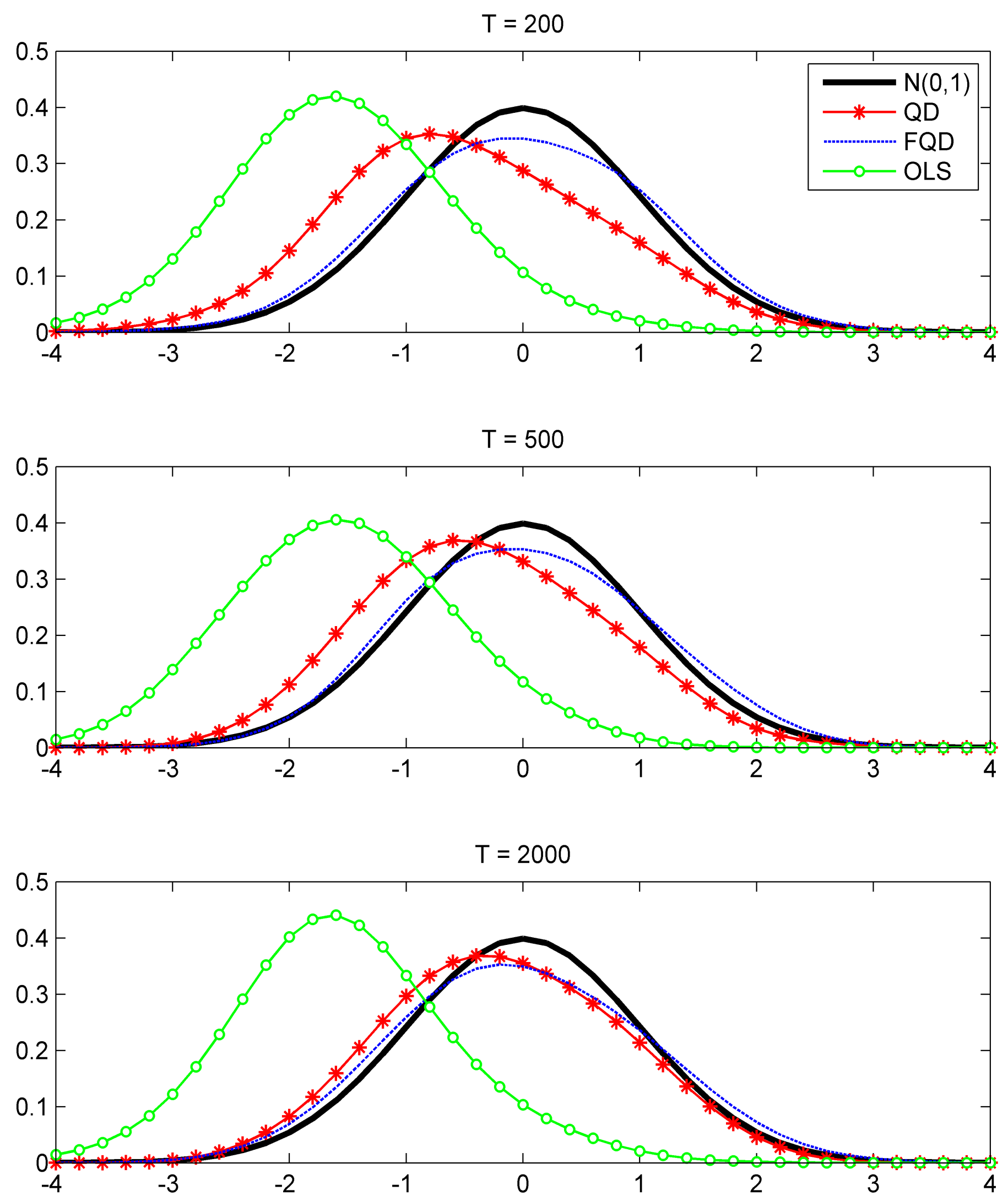

Figure 1: Distribution of the t-statistic for the largest autoregressive root in the intercept-only model with $\alpha^{0}=1$. See Table 1 and the text for more details. 